\title{
Supporting Information for Artificial Intelligence Guided De Novo Molecular Design targeting COVID-19
}

\author{
Srilok Srinivasan ${ }^{1}$, Rohit Batra ${ }^{1}$, Henry Chan ${ }^{1,4}$, Ganesh Kamath ${ }^{2}$, Mathew J. \\ Cherukara $^{1,3}$, and Subramanian K.R.S Sankaranarayanan*1,4 \\ ${ }^{1}$ Center for Nanoscale Materials, Argonne National Laboratory, Lemont, Illinois 60439, \\ United States \\ ${ }^{2}$ Dalzielfiver LLC, 3500 Carlfield St., El Sobrante, CA 94803, United States \\ ${ }^{3}$ Advanced Photon Source, Argonne National Laboratory, Lemont, Illinois 60439, United \\ States \\ ${ }^{4}$ Department of Mechanical and Industrial Engineering, University of Illinois,Chicago, \\ Illinois 60607, United States
}

February 9, 2021 


\section{S 1 Training and Validation of Neural Network Models}
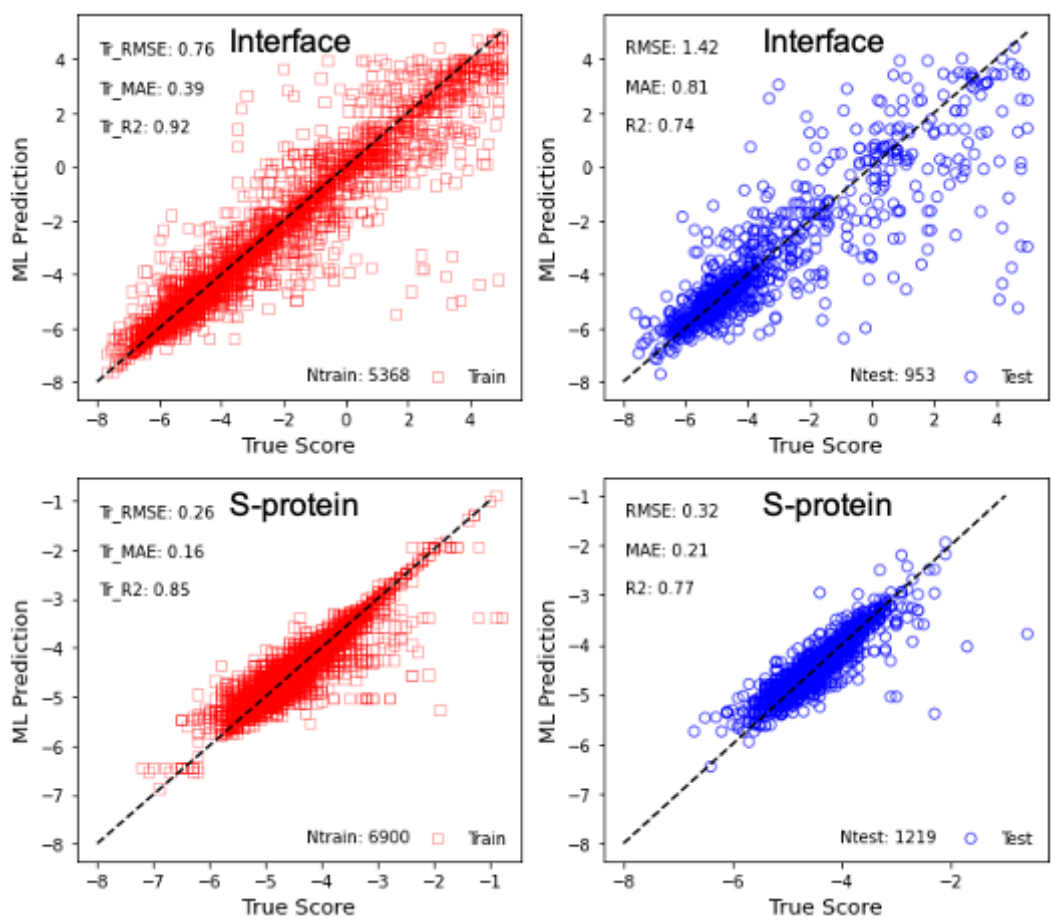

Figure S1: Parity plot of the MTNN Vina score predictions for the S-protein:ACE2 interface (top panel) and the isolated S-protein (bottom panel) systems against those obtained from the Smith dataset. Results for both the training (left panels) and the validation (right panel) set are presented. 

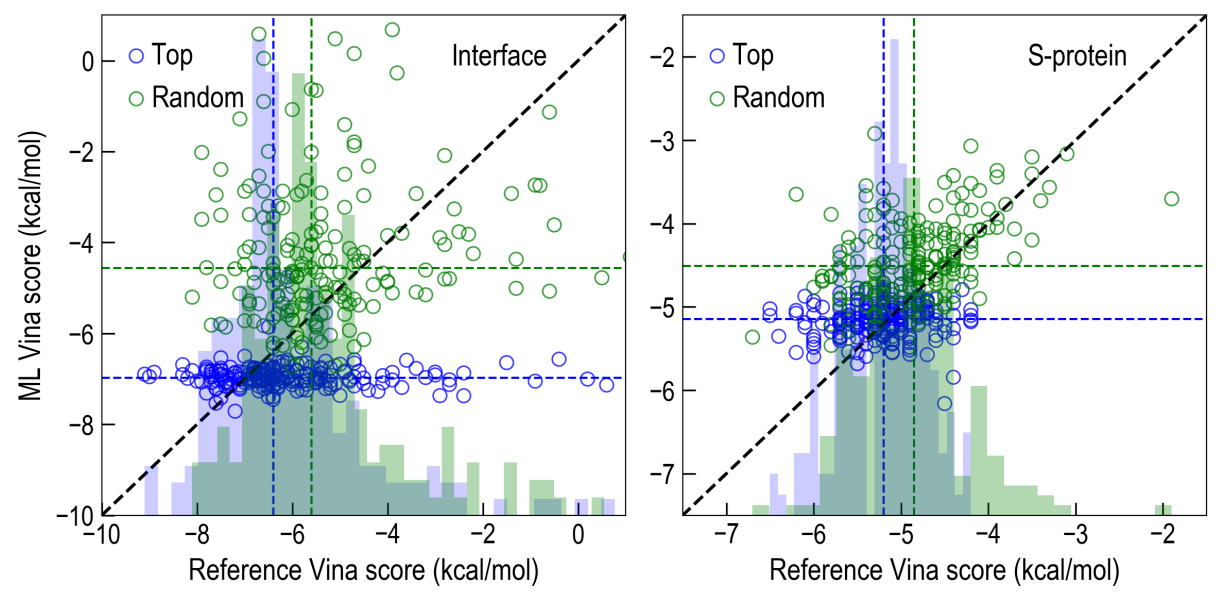

Figure S2: Parity plot of the MTNN Vina score predictions for the S-protein:ACE2 interface (left panel) and the isolated S-protein (right panel) systems against those obtained from the autodock simulations for the identified top (blue) and randomly (green) chosen candidate from the molecules sampled with MCTS using the retrained MTNN (round 2). In each panel, histograms for the top (blue) and randomly (green) chosen candidate molecules illustrate that MTNN models indeed help the MCTS to sample candidates with low Vina scores. This is further supported by the dashed horizontal and vertical lines, which denote the median MTNN prediction and Autodocking score, respectively. We note that 3 of the selected top 200 candidates had greater than 0 vina score (i.e., no binding affinity) for the S-protein:ACE2 interface complex, and are not included in this plot for better readability. These cases highlight limitations of the MTNN model employed and are available in the Supporting files. 


\section{S 2 Training of RNN}
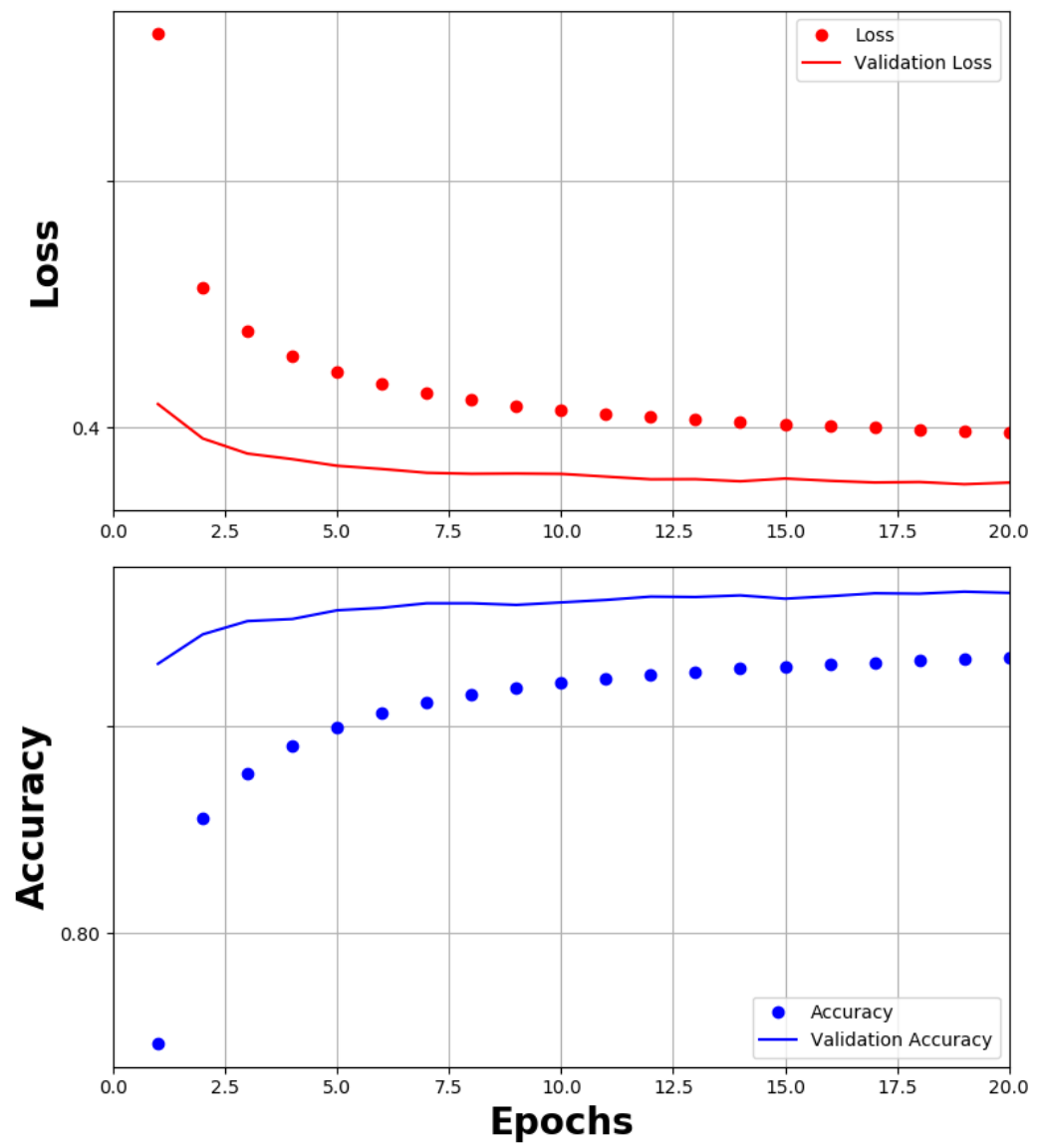

Figure S3: Loss and the accuracy of RNN during the training. The RNN was trained on 945512 SMILES string and the validation set consist of 105057 SMILES string 


\section{S 3 Distribution of binding Vina scores as predicted by MTNN}

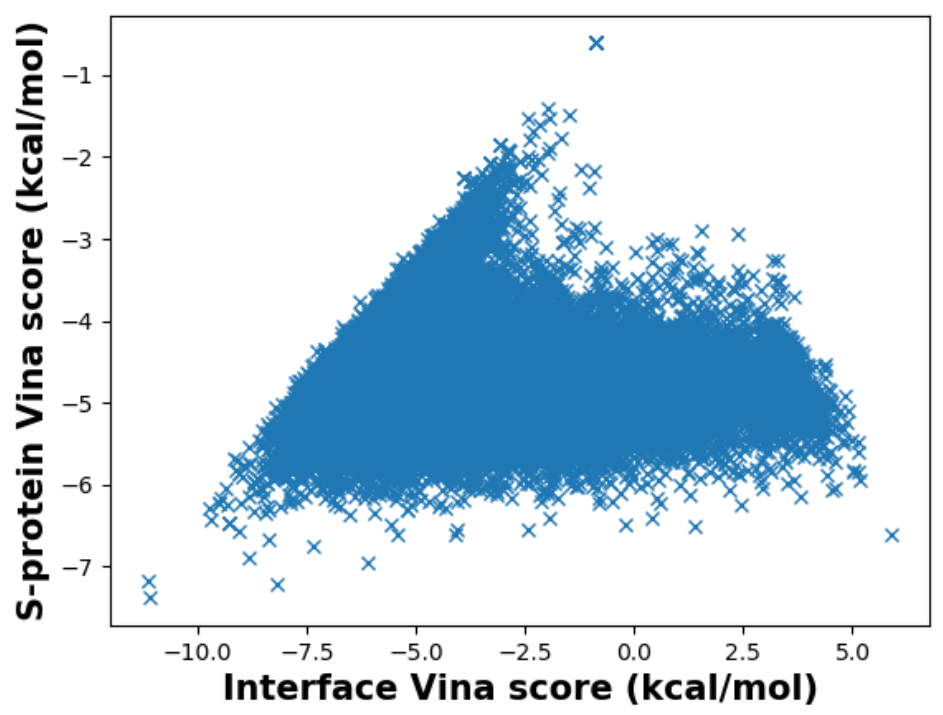

Figure S4: Distribution of the MTNN vina scores sampled by MCTS with the original MTNN (round 1)

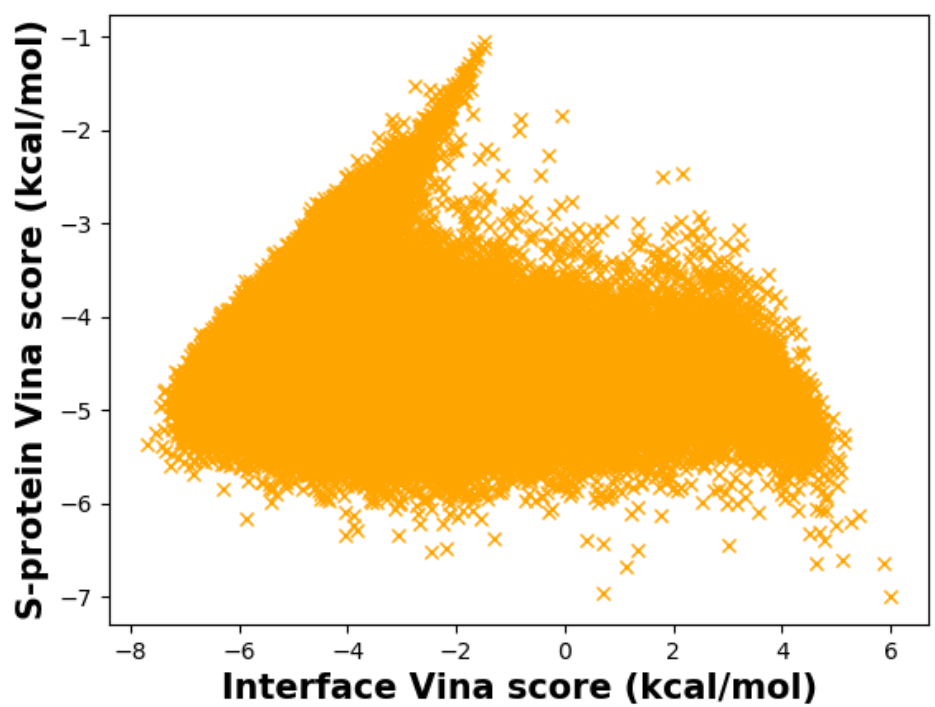

Figure S5: Distribution of the MTNN vina scores sampled by MCTS with improved MTNN (round 2) 
S 4 List of SMILES building block for MCTS sampling

\begin{tabular}{|c|c|c|c|}
\hline$-\&$ & $-[\mathrm{NH}+]$ & $-[\mathrm{SH}-]$ & $-[\mathrm{Fe}+2]$ \\
\hline$-\mathrm{C}$ & $-[\mathrm{S}+]$ & $-[\mathrm{Sn}]$ & $-[\mathrm{Pd}]$ \\
\hline$-\mathrm{O}$ & $-[\mathrm{nH}+]$ & $-[\mathrm{Te}]$ & $-[\mathrm{PH}+]$ \\
\hline$-\mathrm{c}$ & $-[\mathrm{SeH}]$ & $-[\mathrm{Os}]$ & $-[\mathrm{Fe}-2]$ \\
\hline-1 & $-[\mathrm{Br}-]$ & $-[\mathrm{Ru}-]$ & $-[\mathrm{I}+]$ \\
\hline-( & $-[\mathrm{Na}+]$ & $-[\mathrm{Cl}+3]$ & $-[\mathrm{Ru}]$ \\
\hline- ) & $-[\mathrm{n}-]$ & $-[\mathrm{As}]$ & $-[\mathrm{Zn}+2]$ \\
\hline-2 & $-[\mathrm{P} @]$ & -9 & $-[\mathrm{P}-]$ \\
\hline$-[\mathrm{nH}]$ & $-[\mathrm{O}]$ & $-\%$ & $-[\mathrm{NH} 4+]$ \\
\hline$-\mathrm{N}$ & $-[\mathrm{K}+]$ & -0 & $-[\mathrm{Nb}-2]$ \\
\hline$-=$ & $-[\mathrm{Na}]$ & $-[\mathrm{C}+]$ & $-[\mathrm{Pt}-2]$ \\
\hline-3 & $-[\mathrm{Li}+]$ & $-[\mathrm{O}+]$ & $-[\mathrm{Fe}-3]$ \\
\hline-- & $-[\mathrm{Li}]$ & $-[\mathrm{o}+]$ & $-[\mathrm{Al}-3]$ \\
\hline$-\mathrm{n}$ & $-\mathrm{B}$ & $-[\mathrm{N} @ \mathrm{H}+]$ & $-[\mathrm{Cu}-]$ \\
\hline$-[\mathrm{C} @ \mathrm{H}]$ & $-[\mathrm{B}-]$ & - [N@@H+] & $-[\mathrm{Ag}-]$ \\
\hline$-\mathrm{S}$ & $-[\mathrm{OH}+]$ & $-[\mathrm{s}+]$ & $-[\mathrm{Au}-]$ \\
\hline$-[\mathrm{C} @ @ \mathrm{H}]$ & $-[\mathrm{c}+]$ & $-[\mathrm{Hg}]$ & $-[$ As- $]$ \\
\hline-4 & $-[\mathrm{Se}]$ & $-[\mathrm{BiH} 3]$ & $-[\mathrm{Pd}-2]$ \\
\hline$-\mathrm{Cl}$ & $-[\mathrm{I}-]$ & $-[\mathrm{Re}-]$ & $-[\mathrm{Sb}]$ \\
\hline$-o$ & $-[\mathrm{P}+]$ & $-[\mathrm{SH}]$ & $-[\mathrm{CH} 2-]$ \\
\hline$-F$ & $-[\mathrm{CH}-]$ & $-[\mathrm{CH}]$ & $-[\mathrm{Pt}]$ \\
\hline-5 & $-[\mathrm{NH}-]$ & $-[\mathrm{cH}-]$ & $-[\mathrm{Mo}]$ \\
\hline$-\mathrm{Br}$ & $-[\mathrm{Re}]$ & $-[\mathrm{I}]$ & $-[\mathrm{I}+3]$ \\
\hline-6 & $-[\mathrm{PH}]$ & $-[\mathrm{SiH} 2]$ & $-[\mathrm{Br}+2]$ \\
\hline$-\mathrm{s}$ & - [P@@] & $-[\mathrm{C}]$ & $-[\mathrm{I}+2]$ \\
\hline$-\#$ & - [S@@] & $-*$ & $-[\mathrm{Cl}+2]$ \\
\hline$-[\mathrm{C}-]$ & $-[S @+]$ & $-[2 \mathrm{H}]$ & $-[\mathrm{CH} 2+]$ \\
\hline$-[\mathrm{N}+]$ & $-[\mathrm{S} @+]$ & $-[\mathrm{SiH}]$ & $-[\mathrm{Fe}]$ \\
\hline-1 & $-[\mathrm{N} @+]$ & $-[\mathrm{K}]$ & $-[3 \mathrm{H}]$ \\
\hline-1 & $-[\mathrm{N} @ @+]$ & $-[\mathrm{OH}-]$ & $-[\mathrm{Co}]$ \\
\hline$-[\mathrm{Si}]$ & $-[\mathrm{V}]$ & $-[\mathrm{Ca}]$ & $-[\mathrm{Sr}+2]$ \\
\hline-7 & $-[\mathrm{NH} 2+]$ & $-[\mathrm{Cu}]$ & $-[125 \mathrm{I}]$ \\
\hline$-[\mathrm{O}-]$ & $-[\mathrm{CH}+]$ & $-[\mathrm{Mn}]$ & $-[\mathrm{te}]$ \\
\hline$-[\mathrm{C} @]$ & $-[\mathrm{c}-]$ & $-[\mathrm{BH}-]$ & $-[18 \mathrm{~F}]$ \\
\hline$-\mathrm{I}$ & $-[\mathrm{SH}+]$ & $-[\mathrm{BH} 3-]$ & $-[\mathrm{cH}+]$ \\
\hline$-[\mathrm{C} @ @]$ & $-[\mathrm{BH} 2-]$ & $-[\mathrm{Ca}+2]$ & $-[123 \mathrm{I}]$ \\
\hline$-\mathrm{P}$ & $-[\mathrm{S}]$ & $-[\mathrm{W}]$ & $-[\mathrm{Ni}+2]$ \\
\hline$-[\mathrm{n}+]$ & $-[\mathrm{Zn}+]$ & $-[\mathrm{Se}+]$ & $-[\mathrm{Tc}]$ \\
\hline- & $-[\mathrm{se}]$ & $-[\mathrm{N} @]$ & $-[\mathrm{Ni}]$ \\
\hline$-[\mathrm{S} @]$ & $-[\mathrm{S}-]$ & $-[\mathrm{N} @ @]$ & $-[\mathrm{CH} 2]$ \\
\hline-8 & $-[\mathrm{Au}]$ & $-[\mathrm{Se}-]$ & $-[\mathrm{Gd}-4]$ \\
\hline$-[\mathrm{N}-]$ & $-[\mathrm{F}-]$ & $-[\mathrm{Zn}]$ & $-[\mathrm{Gd}-5]$ \\
\hline$-[\mathrm{NH} 3+]$ & $-[\mathrm{Cl}-]$ & $-[11 \mathrm{CH} 3]$ & $-[\mathrm{SiH} 3]$ \\
\hline
\end{tabular}




\section{S 5 Rank ordering of top 200 MCTS sampled molecules}

Table S1: Top 200 candidates sampled my MCTS with improved MTNN model and their S-protein Vina scores

\begin{tabular}{|c|c|}
\hline SMILES & SP-Vina $(\mathrm{kcal} / \mathrm{mol})$ \\
\hline $\mathrm{N} \# \mathrm{Cc} 1 \mathrm{ccc}(\mathrm{Cl}) \mathrm{c}(\mathrm{ONc} 2 \mathrm{cnnn} 2-\mathrm{n} 2 \mathrm{nnn} 2 \mathrm{~F}) \mathrm{c} 1$ & -5.700 \\
\hline $\mathrm{Nc} 1[\mathrm{nH}] \mathrm{c}(=\mathrm{O})[\mathrm{nH}] \mathrm{c} 1 \mathrm{OCc} 1 \mathrm{nsc}(\mathrm{Cn} 2 \mathrm{nnc}(-\mathrm{c} 3 \mathrm{ccccc} 3) \mathrm{c} 2-\mathrm{c} 2 \mathrm{ccco} 2) \mathrm{n} 1$ & -6.000 \\
\hline $\mathrm{O}=\mathrm{C}(\mathrm{Nc} 1 \mathrm{ccc} 2 \mathrm{c}(\mathrm{c} 1) \mathrm{CC}(\mathrm{Oc} 1 \mathrm{cnnn} 1-\mathrm{n} 1 \mathrm{nnnc} 1 \mathrm{~F}) \mathrm{C} 2) \mathrm{c} 1 \mathrm{nc} 2 \mathrm{ccccc} 2[\mathrm{nH}] 1$ & -5.400 \\
\hline $\mathrm{Nc1}[\mathrm{nH}] \mathrm{c}(=\mathrm{O})[\mathrm{nH}] \mathrm{c1}-\mathrm{c} 1 \mathrm{nsc}(\mathrm{C}[\mathrm{C} @ @ \mathrm{H}] 2 \mathrm{C}(=\mathrm{O}) \mathrm{NCC}[\mathrm{C} @ @ \mathrm{H}] 2 \mathrm{O}) \mathrm{n} 1$ & -4.900 \\
\hline $\mathrm{Nc} 1[\mathrm{nH}] \mathrm{c}(=\mathrm{O})[\mathrm{nH}] \mathrm{c} 1-\mathrm{c} 1 \operatorname{noc}(\mathrm{NC} 2=\mathrm{N}[\mathrm{C} @ \mathrm{H}](\mathrm{S}(=\mathrm{O})(=\mathrm{O}) \mathrm{Cc} 3 \mathrm{ccccc} 3) \mathrm{C} 2) \mathrm{n} 1$ & -5.500 \\
\hline $\mathrm{Nc} 1[\mathrm{nH}] \mathrm{c}(=\mathrm{O})[\mathrm{nH}] \mathrm{c} 1 \mathrm{OCc} 1 \mathrm{nc}(-\mathrm{c} 2 \mathrm{cnc}(\mathrm{C} 3(\mathrm{C}(=\mathrm{O}) \mathrm{O}) \mathrm{CCC} 3) \mathrm{c}(\mathrm{Cl}) \mathrm{c} 2) \mathrm{no} 1$ & -5.300 \\
\hline$[\mathrm{NH} 2+][\mathrm{C} @ \mathrm{H}]([\mathrm{NH}+][\mathrm{C} @ \mathrm{H}](\mathrm{c} 1 \mathrm{ccco} 1)[\mathrm{C} @ @ \mathrm{H}](\mathrm{CC}(=\mathrm{O}) \mathrm{O}) \mathrm{C}(\mathrm{F})(\mathrm{F}) \mathrm{F}) \mathrm{c} 1 \mathrm{ncno1}$ & -4.900 \\
\hline $\mathrm{Nc} 1[\mathrm{nH}] \mathrm{c}(=\mathrm{O})[\mathrm{nH}] \mathrm{c} 1 \mathrm{OC}[\mathrm{C} @ \mathrm{H}](\mathrm{CCC}(=\mathrm{O}) \mathrm{O}) \mathrm{NC}(=\mathrm{O}) \mathrm{Nc1cc}(\mathrm{Cl}) \mathrm{cc}(\mathrm{Cl}) \mathrm{c1}$ & -4.900 \\
\hline$[\mathrm{NH} 2+][\mathrm{C} @ \mathrm{H}](\mathrm{N}[\mathrm{C} @ @ \mathrm{H}](\mathrm{Cc} 1 \mathrm{ccncc} 1) \mathrm{c} 1 \mathrm{ccccc} 1) \mathrm{c} 1 \mathrm{nc} 2 \mathrm{ncccc} 2 \mathrm{o} 1$ & -5.000 \\
\hline $\mathrm{Nc} 1[\mathrm{nH}] \mathrm{c}(=\mathrm{O})[\mathrm{nH}] \mathrm{c} 1 \mathrm{OCc} 1 \mathrm{nnn}(\mathrm{C}[\mathrm{C} @ \mathrm{H}](\mathrm{CC}(=\mathrm{O}) \mathrm{O}) \mathrm{c} 2 \mathrm{ccccc} 2 \mathrm{Cl}) \mathrm{n} 1$ & -5.500 \\
\hline Fc1nnnn1-n1nnc2cc(NCc3nnc[n-]3) ccc21 & -5.400 \\
\hline $\mathrm{C}[\mathrm{C} @ @] 1(\mathrm{c} 2 \mathrm{ccco} 2) \mathrm{O}[\mathrm{C} @ @ \mathrm{H}] 2 \mathrm{~S}[\mathrm{C} @ \mathrm{H}](\mathrm{Oc} 3[\mathrm{nH}] \mathrm{c}(=\mathrm{O})[\mathrm{nH}] \mathrm{c} 3 \mathrm{~N}) \mathrm{C}(=\mathrm{O}) \mathrm{N} 21$ & -5.100 \\
\hline $\mathrm{O}=\mathrm{C}(\mathrm{NCc} 1 \mathrm{ncoc} 1 \mathrm{O}) \mathrm{n} 1 \mathrm{cc}(\mathrm{C}(\mathrm{F})(\mathrm{F}) \mathrm{F}) \mathrm{nn} 1$ & -5.100 \\
\hline $\mathrm{O}=\mathrm{C}(\mathrm{CSc} 1 \mathrm{nc} 2 \operatorname{ccccc} 2 \mathrm{o} 1) \mathrm{Nc} 1 \mathrm{ccc}(\mathrm{C}(=\mathrm{O}) \mathrm{c} 2 \operatorname{ccc}(\mathrm{O}) \mathrm{cc} 2) \mathrm{o} 1$ & -5.000 \\
\hline $\mathrm{Nc1}[\mathrm{nH}] \mathrm{nnc1CNC}(=\mathrm{O}) \mathrm{C}(\mathrm{Nc} 1 \mathrm{ccccc} 1) \mathrm{c} 1 \mathrm{ccccc} 1$ & -5.700 \\
\hline $\mathrm{Nc} 1[\mathrm{nH}] \mathrm{c} 2 \mathrm{nc}(-\mathrm{c} 3 \mathrm{nc}(\mathrm{O}) \mathrm{cc}(=\mathrm{O}) \mathrm{o} 3) \mathrm{nnc} 2 \mathrm{c} 1-\mathrm{c} 1 \mathrm{ccc}(\mathrm{NC}(=\mathrm{O}) \mathrm{C} 2 \mathrm{CCOC} 2) \mathrm{cc} 1$ & -4.200 \\
\hline$[\mathrm{NH} 2+][\mathrm{C} @ \mathrm{H}](\mathrm{Cn} 1 \mathrm{nnn}(\mathrm{N}(\mathrm{CCF}) \mathrm{Cc} 2 \mathrm{ccccc} 2) \mathrm{c} 1=\mathrm{O}) \mathrm{c} 1 \mathrm{ncc}[\mathrm{nH}] 1$ & -4.900 \\
\hline $\mathrm{Nc} 1[\mathrm{nH}] \mathrm{c}(=\mathrm{O})[\mathrm{nH}] \mathrm{c} 1 \mathrm{OCc} 1 \mathrm{nc} 2 \mathrm{nn}[\mathrm{nH}] \mathrm{c} 2 \mathrm{c} 2 \mathrm{ccccc} 12$ & -5.600 \\
\hline $\mathrm{Nc} 1[\mathrm{nH}] \mathrm{c}(=\mathrm{O})[\mathrm{nH}] \mathrm{c} 1-\mathrm{c} 1 \mathrm{nsnc} 1 \mathrm{OCC} / \mathrm{C}=\mathrm{C} / \mathrm{CCC}(=\mathrm{O}) \mathrm{NO}$ & -5.100 \\
\hline $\mathrm{Nc} 1[\mathrm{nH}] \mathrm{c}(=\mathrm{O})[\mathrm{nH}] \mathrm{c} 1 \mathrm{OCC}(=\mathrm{O}) \mathrm{COC}(=\mathrm{O}) \mathrm{c} 1 \mathrm{ccccc} 1 \mathrm{~F}$ & -5.100 \\
\hline $\mathrm{O}[\mathrm{C} @ \mathrm{H}] 1 \mathrm{C}[\mathrm{C} @](\mathrm{O})([\mathrm{C} @ \mathrm{H}](\mathrm{c} 2 \mathrm{ccsc} 2) \mathrm{n} 2 \mathrm{nnn} c 2-\mathrm{n} 2 \mathrm{nnn} c 2 \mathrm{~F}) \mathrm{C} 1$ & -5.200 \\
\hline $\mathrm{O}=\mathrm{C}(\mathrm{Nc} 1 \mathrm{ccnc}(\mathrm{Oc} 2 \mathrm{ccnc} 3 \mathrm{nnn}(-\mathrm{n} 4 \mathrm{nnnc} 4 \mathrm{~F}) \mathrm{c} 23) \mathrm{c} 1 \mathrm{C}(\mathrm{F})(\mathrm{F}) \mathrm{F}) \mathrm{c} 1 \mathrm{cccs} 1$ & -5.500 \\
\hline $\mathrm{Nc} 1[\mathrm{nH}] \mathrm{c}(=\mathrm{O})[\mathrm{nH}] \mathrm{c} 1 \mathrm{OCc} 1 \mathrm{nsc} 2 \mathrm{sc} 3 \mathrm{ccccc} 3 \mathrm{c} 12$ & -5.200 \\
\hline $\mathrm{Cn} 1 \mathrm{nnn}(-\mathrm{n} 2 \mathrm{nnnn} 2 \mathrm{~F}) \mathrm{c} 1=\mathrm{NNC}(=\mathrm{O})[\mathrm{C} @ \mathrm{H}](\mathrm{Cc} 1 \mathrm{ccccc} 1) \mathrm{NC}(=\mathrm{O}) \mathrm{c} 1 \mathrm{ccccc} 1$ & -5.800 \\
\hline $\mathrm{O}=\mathrm{C}(\mathrm{NC}[\mathrm{NH}+]=\mathrm{c} 1 \mathrm{c} 2 \mathrm{cc}(\mathrm{F}) \mathrm{ccc} 2 \mathrm{nnn} 1-\mathrm{n} 1 \mathrm{nnnnc} 1 \mathrm{~F}) \mathrm{c} 1 \mathrm{ccccc} 1$ & -6.400 \\
\hline$[\mathrm{NH} 2+][\mathrm{C} @ \mathrm{H}]([\mathrm{NH}+][\mathrm{C} @ @ \mathrm{H}] 1 \mathrm{OCCCCc} 2 \mathrm{c} 1 \mathrm{ccn} 2-\mathrm{c} 1 \mathrm{ccccc} 1) \mathrm{c} 1 \mathrm{ncno1}$ & -5.300 \\
\hline $\mathrm{N} \# \mathrm{C}[\mathrm{C} @ @ \mathrm{H}](\mathrm{COc} 1 \mathrm{cnon} 1) \mathrm{NC} 1=\mathrm{C}(\mathrm{C}(=\mathrm{O}) \mathrm{O}) \mathrm{C}[\mathrm{C} @ \mathrm{H}] 2 \mathrm{CCC}[\mathrm{C} @ \mathrm{H}] 12$ & -5.100 \\
\hline $\mathrm{Nc} 1[\mathrm{nH}] \mathrm{c}(=\mathrm{O})[\mathrm{nH}] \mathrm{c} 1 \mathrm{OCc} 1 \mathrm{n}[\mathrm{nH}] \mathrm{cc} 1-\mathrm{n} 1 \mathrm{nc} 2 \mathrm{ccccc} 2 \mathrm{c} 1 \mathrm{~N} 1 \mathrm{CCC}[\mathrm{C} @ \mathrm{H}](\mathrm{C}(=\mathrm{O}) \mathrm{O}) \mathrm{C} 1$ & -5.600 \\
\hline $\mathrm{Nc} 1[\mathrm{nH}] \mathrm{c}(=\mathrm{O})[\mathrm{nH}] \mathrm{c} 1 \mathrm{OC}[\mathrm{C} @ \mathrm{H}](\mathrm{N}) \mathrm{CNC}(=\mathrm{O}) \mathrm{c} 1 \mathrm{ccc}(-\mathrm{c} 2 \mathrm{ccccc} 2) \mathrm{cc} 1$ & -5.400 \\
\hline Fc1nnnn1-n1nnc2cc(NCc3cc(-c4ccccc4)[nH]c3-c3ccccc3) $\operatorname{ccc} 21$ & -6.000 \\
\hline$[\mathrm{NH} 2+][\mathrm{C} @ @ \mathrm{H}](\mathrm{c} 1 \mathrm{ncns} 1) \mathrm{c} 1 \mathrm{cc} 2 \mathrm{occn} 2 \mathrm{c} 1 \mathrm{C}[\mathrm{NH}+] 1 \mathrm{Cc} 2 \mathrm{ccccc} 2 \mathrm{C} 1=\mathrm{O}$ & -5.000 \\
\hline$[\mathrm{NH} 2+] \mathrm{C}(\mathrm{N}[\mathrm{C} @ @ \mathrm{H}](\mathrm{CO}) \mathrm{C}(=\mathrm{O}) \mathrm{O}) \mathrm{c} 1 \mathrm{ccc}(\mathrm{F}) \mathrm{cc} 1$ & -4.200 \\
\hline $\mathrm{Nc} 1[\mathrm{nH}] \mathrm{c}(=\mathrm{O})[\mathrm{nH}] \mathrm{c} 1 \mathrm{OC}[\mathrm{C} @ \mathrm{H}]([\mathrm{O}-])[\mathrm{C} @ @ \mathrm{H}](\mathrm{N})[\mathrm{C} @ @ \mathrm{H}](\mathrm{O}) \mathrm{C}(\mathrm{F})(\mathrm{F}) \mathrm{F}$ & -4.700 \\
\hline $\mathrm{Nc} 1[\mathrm{nH}] \mathrm{c}(=\mathrm{O})[\mathrm{nH}] \mathrm{c} 1 \mathrm{OC} 1 \mathrm{c} 2 \operatorname{ccccc} 2 \mathrm{C}[\mathrm{SH}] 1 \mathrm{C} 1=\mathrm{NS}(=\mathrm{O})(=\mathrm{O}) \mathrm{N} 1 \mathrm{C} 1 \mathrm{CC} 1$ & -5.400 \\
\hline $\mathrm{Nc} 1[\mathrm{nH}] \mathrm{nnn} 1-\mathrm{c} 1 \mathrm{cn}(\mathrm{C} 2(\mathrm{C}(=\mathrm{O}) \mathrm{O}) \mathrm{CCCCCCC}(=\mathrm{O}) \mathrm{O} 2) \mathrm{s} 1$ & -5.000 \\
\hline Oc1ccc $(\mathrm{Cc} 2 \mathrm{noc}([\mathrm{C} @ @ \mathrm{H}](\mathrm{O}) \mathrm{CNCc} 3 \mathrm{cnnn} 3-\mathrm{n} 3 \mathrm{nnnn} 3 \mathrm{~F}) \mathrm{n} 2) \mathrm{c}(\mathrm{C} 2 \mathrm{CC} 2) \mathrm{c} 1$ & -5.100 \\
\hline $\mathrm{Nc} 1[\mathrm{nH}] \mathrm{c}(=\mathrm{O})[\mathrm{nH}] \mathrm{c} 1 \mathrm{O}[\mathrm{CH}] \mathrm{c} 1 \mathrm{ccccc} 1 \mathrm{CNS}(=\mathrm{O})(=\mathrm{O}) \mathrm{C} 1 \mathrm{CC} 1$ & -4.900 \\
\hline$[\mathrm{NH} 2+][\mathrm{C} @ \mathrm{H}](\mathrm{C}[\mathrm{C} @ \mathrm{H}] 1 \mathrm{COc} 2 \mathrm{ccccc} 2 \mathrm{C} 1) \mathrm{c} 1 \mathrm{ncn} 2 \mathrm{ncnc} 2 \mathrm{n} 1$ & -5.100 \\
\hline$[\mathrm{NH} 2+][\mathrm{C} @ @ \mathrm{H}](\mathrm{c} 1 \mathrm{ncc}[\mathrm{nH}] 1)[\mathrm{C} @ \mathrm{H}] 1 \mathrm{c} 2[\mathrm{nH}] \mathrm{nnc} 2 \mathrm{C}[\mathrm{C} @ @ \mathrm{H}] 2 \mathrm{c} 3 \mathrm{ccccc} 3 \mathrm{C}[\mathrm{C} @ @ \mathrm{H}] 21$ & -5.000 \\
\hline $\mathrm{O}=\mathrm{C}(\mathrm{Nc} 1 \mathrm{ccc} 2 \mathrm{c}(\mathrm{c} 1) \mathrm{nnn} 2-\mathrm{c} 1 \mathrm{cnnn} 1-\mathrm{n} 1 \mathrm{nnnn} 1 \mathrm{~F}) \mathrm{c} 1 \mathrm{cccs} 1$ & -5.700 \\
\hline $\mathrm{Nc} 1 \mathrm{c}([\mathrm{C} @ \mathrm{H}]([\mathrm{NH} 2+]) \mathrm{C} 2 \mathrm{CCC}[\mathrm{C} @ @ \mathrm{H}] 2 \mathrm{C}(=\mathrm{O})[\mathrm{O}-]) \mathrm{ncn} 1 \mathrm{Cl}$ & -4.200 \\
\hline $\mathrm{O}=\mathrm{C}(\mathrm{Nc} 1 \mathrm{ccc}(\mathrm{C}(\mathrm{Oc} 2 \mathrm{nnn}[\mathrm{nH}] 2) \mathrm{n} 2 \mathrm{nnnc} 2 \mathrm{~F}) \mathrm{cc} 1) \mathrm{c} 1 \mathrm{ccccc} 1 \mathrm{Cl}$ & -6.200 \\
\hline$[\mathrm{NH} 2+][\mathrm{C} @ @ \mathrm{H}](\mathrm{c} 1 \mathrm{ncc}[\mathrm{nH}] 1)[\mathrm{C} @ @ \mathrm{H}] 1 \mathrm{C}(=\mathrm{O}) \mathrm{N} 2 \mathrm{C}(=\mathrm{O}) \mathrm{N}=\mathrm{C} 2 \mathrm{Sc} 2 \mathrm{nn}[\mathrm{nH}] \mathrm{c} 21$ & -4.900 \\
\hline $\mathrm{CP}(=\mathrm{O})([\mathrm{O}-]) \mathrm{OCc} 1 \mathrm{c}([\mathrm{C} @ \mathrm{H}]([\mathrm{NH} 2+]) \mathrm{c} 2 \mathrm{ccccc} 2) \mathrm{nc} 2 \mathrm{ncccn} 12$ & -4.800 \\
\hline $\mathrm{Nc} 1[\mathrm{nH}] \mathrm{c}(=\mathrm{O})[\mathrm{nH}] \mathrm{c} 1 \mathrm{O}[\mathrm{C} @ \mathrm{H}] 1 \mathrm{SN}[\mathrm{C} @ @ \mathrm{H}] 2 \mathrm{c} 3 \mathrm{ccc}(\mathrm{Cl}) \mathrm{cc} 3[\mathrm{C} @ \mathrm{H}] 12$ & -4.900 \\
\hline $\mathrm{Nc} 1[\mathrm{nH}] \mathrm{c}(=\mathrm{O})[\mathrm{nH}] \mathrm{c} 1-\mathrm{c} 1 \mathrm{nc} 2 \mathrm{c}(\mathrm{s} 1) \mathrm{CCCc} 1 \mathrm{ccc}(\mathrm{S}(=\mathrm{O})(=\mathrm{O}) \mathrm{Nc} 3 n n n[\mathrm{nH}] 3) \mathrm{cc} 1-2$ & -6.000 \\
\hline$[\mathrm{NH} 2+] \mathrm{Cc} 1 \mathrm{cnnn}(\mathrm{Oc} 2 \mathrm{c}(\mathrm{O}) \operatorname{ccc} 3 \operatorname{cccc}(\mathrm{O}) \mathrm{c} 23) \mathrm{c} 1$ & -5.100 \\
\hline Fc1nnnn1-n1nncc1-n1nnc(N=[NH+]Cc2ccc $(\mathrm{OCc} 3 \operatorname{cccs} 3) \operatorname{cc} 2) n 1$ & -5.700 \\
\hline
\end{tabular}


$\mathrm{Nc} 1[\mathrm{nH}] \mathrm{c}(=\mathrm{O})[\mathrm{nH}] \mathrm{c} 1 \mathrm{C}=\mathrm{C} 1 \mathrm{~N}[\mathrm{~N}-] \mathrm{N}(\mathrm{c} 2 \mathrm{c}(\mathrm{F}) \operatorname{cccc} 2 \mathrm{C}(\mathrm{F})(\mathrm{F}) \mathrm{F}) \mathrm{C} 1=\mathrm{O}$

$[\mathrm{NH} 2+][\mathrm{C} @ @ \mathrm{H}](\mathrm{c} 1 \mathrm{nc} 2 \mathrm{ncccc} 2 \mathrm{o} 1) \mathrm{c} 1 \mathrm{nncs} 1$

$\mathrm{Nc} 1[\mathrm{nH}] \mathrm{c}(=\mathrm{O})[\mathrm{nH}] \mathrm{c} 1-\mathrm{c} 1 \mathrm{csc} 2 \mathrm{nnnc}(\mathrm{NSc} 3 \mathrm{nccs} 3) \mathrm{c} 12$

$\mathrm{NCc} 1 \mathrm{cc}(-\mathrm{c} 2 \mathrm{nnn}([\mathrm{C} @ @ \mathrm{H}](\mathrm{CCC}(=\mathrm{O}) \mathrm{O}) \mathrm{C}(=\mathrm{O}) \mathrm{CO}) \mathrm{n} 2) \mathrm{c}[\mathrm{nH}] \mathrm{c} 1=\mathrm{O}$

$[\mathrm{NH} 2+][\mathrm{C} @ @ \mathrm{H}](\mathrm{c} 1 \mathrm{nc} 2 \mathrm{ccsc} 2[\mathrm{nH}] 1)[\mathrm{C} @ @ \mathrm{H}] 1 \mathrm{Oc} 2 \mathrm{c}([\mathrm{nH}] \mathrm{c} 3 \mathrm{ccccc} 23)[\mathrm{C} @ \mathrm{H}](\mathrm{O})[\mathrm{C} @ @ \mathrm{H}] 1 \mathrm{O}$

$[\mathrm{NH} 2+][\mathrm{C} @ @ \mathrm{H}](\mathrm{c} 1 \mathrm{nc} 2 \mathrm{ncncc} 2 \mathrm{~s} 1) \mathrm{n} 1[\mathrm{nH}+] \mathrm{c} 2[\mathrm{nH}] \mathrm{c} 3 \mathrm{ncccc} 3 \mathrm{nc} 1-2$

$[\mathrm{NH} 2+][\mathrm{C} @ @ \mathrm{H}](\mathrm{c} 1 \mathrm{nc} 2 \mathrm{cn}[\mathrm{nH}] \mathrm{c} 2[\mathrm{nH}] 1) \mathrm{C} 1[\mathrm{C} @ \mathrm{H}] 2 \mathrm{CCC}[\mathrm{C} @ \mathrm{H}] 1 \mathrm{CS}(=\mathrm{O})(=\mathrm{O}) \mathrm{C} 2$

Fc1nnnn1-n1nncc1N[C@H]1[CH]CNC[C@@H]1Cc1ccc(Cl)cc1

$\mathrm{Nc} 1[\mathrm{nH}] \mathrm{c}(=\mathrm{O})[\mathrm{nH}] \mathrm{c} 1-\mathrm{c} 1 \mathrm{n}[\mathrm{nH}] \mathrm{cc} 1 \mathrm{NS}(=\mathrm{O})(=\mathrm{O}) \mathrm{C} 1 \mathrm{CCOCC} 1$

Fc1nnnn1-n1nncc1OCCc1noc2cc $(\mathrm{OCc} 3 \mathrm{c}(\mathrm{Cl}) \mathrm{cccc} 3 \mathrm{Cl}) \mathrm{ccc} 12$

$\mathrm{Nc} 1[\mathrm{nH}] \mathrm{nc}([\mathrm{C} @ @ \mathrm{H}] 2 \mathrm{C}[\mathrm{C} @ @ \mathrm{H}] 3 \mathrm{CC}[\mathrm{C} @ \mathrm{H}] 3 \mathrm{~N} 2) \mathrm{c} 1-\mathrm{c} 1 \mathrm{nnc}[\mathrm{nH}] 1$

$[\mathrm{NH} 2+][\mathrm{C} @ @ H](\mathrm{c} 1 \mathrm{nc}(\mathrm{Br}) \mathrm{no1})[\mathrm{C} @ \mathrm{H}](\mathrm{c} 1 \mathrm{ccccc} 1) \mathrm{c} 1 \mathrm{c}[\mathrm{nH}] \mathrm{c} 2 \mathrm{ccccc} 12$

Fc1ccc(-n2nncc2CO[C@@H](Nc2cnnn2-n2nnnc2F)c2ccccc2)cc1

$[\mathrm{NH} 2+][\mathrm{C} @ @ \mathrm{H}](\mathrm{c} 1 \mathrm{nc} 2 \mathrm{ncccc} 2[\mathrm{nH}] 1)[\mathrm{C} @ \mathrm{H}](\mathrm{Oc} 1 \mathrm{ccc} 2 \mathrm{ccccc} 2 \mathrm{c} 1) \mathrm{c} 1 \mathrm{ccccc} 1$

$[\mathrm{NH} 2+][\mathrm{C} @ @ \mathrm{H}](\mathrm{c} 1 \mathrm{ncco} 1) \mathrm{C}(\mathrm{NC} 1 \mathrm{COC} 1) \mathrm{c} 1 \mathrm{ccccc} 1-\mathrm{c} 1 \mathrm{ccccc} 1$

$[\mathrm{NH} 2+][\mathrm{C} @ @ \mathrm{H}] 1 \mathrm{C}=\mathrm{Nc} 2 \mathrm{nnc}(-\mathrm{c} 3 \mathrm{ccc}(\mathrm{Oc} 4 \mathrm{ccc}(\mathrm{F}) \mathrm{cc} 4) \mathrm{nc} 3) \mathrm{n} 21$

$\mathrm{Nc1}[\mathrm{nH}] \mathrm{nnc1}-\mathrm{c} 1 \mathrm{cccnc1N}[\mathrm{C} @ @ \mathrm{H}] 1[\mathrm{C} @ \mathrm{H}] 2 \mathrm{CC}[\mathrm{C} @ @ \mathrm{H}] 1[\mathrm{C} @ @ \mathrm{H}] 2 \mathrm{C}(=\mathrm{O}) \mathrm{O}$

$[\mathrm{NH} 2+][\mathrm{C} @ @ \mathrm{H}](\mathrm{c} 1 \mathrm{ncns} 1) \mathrm{C} 1 \mathrm{Cc} 2 \mathrm{ncnc} 2 \mathrm{NC} 1=\mathrm{O}$

$[\mathrm{NH} 2+][\mathrm{C} @ \mathrm{H}](\mathrm{Cn} 1 \mathrm{cc}[\mathrm{nH}+] \mathrm{c} 1 \mathrm{Nc} 1 \mathrm{ccccc} 1) \mathrm{c} 1 \mathrm{nc} 2 \mathrm{nccc}[\mathrm{c}-] 2 \mathrm{n} 1$

$\mathrm{Nc} 1[\mathrm{nH}] \mathrm{nnc} 1 \mathrm{C}=\mathrm{NNC}(=\mathrm{O})[\mathrm{C} @ @ \mathrm{H}] 1 \mathrm{CCCO} 1$

$\mathrm{Nc} 1[\mathrm{nH}] \mathrm{c}(=\mathrm{O})[\mathrm{nH}] \mathrm{c} 1 \mathrm{O}[\mathrm{CH}] \mathrm{c} 1 \mathrm{ccc}(\mathrm{N}=\mathrm{Nc} 2 \mathrm{ncccc} 2 \mathrm{O}) \mathrm{cc} 1$

Fc1cc(Oc2 $2 c c c c 2) c c 2 c 1 n n n 2-n 1 n n n c 1 F$

$\mathrm{Nc} 1[\mathrm{nH}] \mathrm{c}(=\mathrm{O})[\mathrm{nH}] \mathrm{c} 1-\mathrm{c} 1 \mathrm{noc}([\mathrm{C} @ @ \mathrm{H}] 2 \mathrm{c} 3 \mathrm{ccsc} 3-\mathrm{c} 3 \mathrm{nnnn} 32) \mathrm{n} 1$

Fc1nnnn1-n1nnc(COc2cnc(OCc3ccc4ccccc4n3)nc2)n1

$\mathrm{Nc} 1[\mathrm{nH}] \mathrm{nnc} 1 \mathrm{CCCOC}(=\mathrm{O}) \mathrm{c} 1 \mathrm{c}(\mathrm{Cl}) \mathrm{cccc} 1 \mathrm{Cl}$

$\mathrm{NC}(=\mathrm{O}) \mathrm{c} 1 \mathrm{ccn} 2 \mathrm{c}(\mathrm{COc} 3[\mathrm{nH}] \mathrm{c}(=\mathrm{O})[\mathrm{nH}] \mathrm{c} 3 \mathrm{~N}) \mathrm{ncc} 2 \mathrm{c} 1$

$\mathrm{O}=\mathrm{c} 1[\mathrm{nH}] \mathrm{c} 2 \operatorname{ccccc} 2 \mathrm{nc} 1 \mathrm{Oc} 1 \mathrm{cccc}(\mathrm{Oc} 2 \mathrm{c}(\mathrm{O})$ noc $2 \mathrm{O}) \mathrm{c} 1$

$\mathrm{O}=\mathrm{S}(=\mathrm{O})(\mathrm{CCOCc} 1 \mathrm{ccccc} 1) \mathrm{Nc} 1 \mathrm{cnnn} 1-\mathrm{n} 1 \mathrm{nnnn} 1 \mathrm{~F}$

$\mathrm{O}=\mathrm{c} 1 \mathrm{cc}(\mathrm{NCc} 2 \mathrm{ccccc} 2) \mathrm{n}(-\mathrm{n} 2 \mathrm{nnnnc} 2 \mathrm{~F}) \mathrm{nn} 1$

$\mathrm{N} \# \mathrm{C}[\mathrm{C} @ \mathrm{H}](\mathrm{CNC}(=\mathrm{O}) \mathrm{C} 1=\mathrm{CC}=\mathrm{CCC} 1) \mathrm{Nc} 1 \mathrm{cnnn} 1-\mathrm{n} 1 \mathrm{nnnnc} 1 \mathrm{~F}$

$\mathrm{Nc} 1[\mathrm{nH}] \mathrm{c}(=\mathrm{O})[\mathrm{nH}] \mathrm{c} 1 \mathrm{OCC} 1 \mathrm{cccc} 2 \mathrm{c} 1 \mathrm{OCCO} 2$

$\mathrm{Nc} 1[\mathrm{nH}] \mathrm{c}(=\mathrm{O})[\mathrm{nH}] \mathrm{c1}-\mathrm{c} 1 \mathrm{noc}(-\mathrm{c} 2 \mathrm{cc} 3 \mathrm{cc}(\mathrm{Cl}) \mathrm{ccc} 3[\mathrm{nH}] \mathrm{c} 2=\mathrm{O}) \mathrm{n} 1$

$\mathrm{Nc} 1[\mathrm{nH}] \mathrm{c}(=\mathrm{O})[\mathrm{nH}] \mathrm{c} 1 \mathrm{O}[\mathrm{C} @ \mathrm{H}] 1 \mathrm{C}[\mathrm{C} @ @ \mathrm{H}] 2 \mathrm{OC}[\mathrm{C} @ \mathrm{H}] 2 \mathrm{O} 1$

Fc1nnnn1-n1nnc2nc(COc3cccc3) $\operatorname{ccc} 21$

Fc1nnnn1-c1nnc(NCc2 2 cccc2)s1

$\mathrm{Nc} 1[\mathrm{nH}] \mathrm{c}(=\mathrm{O})[\mathrm{nH}] \mathrm{c1}-\mathrm{c} 1 \mathrm{c}[\mathrm{nH}] \mathrm{c} 2 \mathrm{c}(\mathrm{Cl}) \mathrm{ncc}(\mathrm{O}[\mathrm{C} @ @ \mathrm{H}] 3 \mathrm{CCNC} 3) \mathrm{c} 12$

$\mathrm{Nc} 1[\mathrm{nH}] \mathrm{c}(=\mathrm{O})[\mathrm{nH}] \mathrm{c} 1 \mathrm{O}[\mathrm{C} @ \mathrm{H}] 1 \mathrm{C}[\mathrm{C} @ @ \mathrm{H}] 2 \mathrm{OC}[\mathrm{C} @ @ \mathrm{H}] 1[\mathrm{C} @ @ \mathrm{H}] 2 \mathrm{CSc} 1 \mathrm{nc}(-\mathrm{c} 2 \mathrm{ccccc} 2) \mathrm{no} 1$

Fc1nnnn1-n1nnc(SONc2nsnc2NCCCn2cnnc2)n1

$\mathrm{Nc} 1[\mathrm{nH}] \mathrm{c}(=\mathrm{O})[\mathrm{nH}] \mathrm{c} 1 \mathrm{OC}[\mathrm{C} @ \mathrm{H}](\mathrm{CO}) \mathrm{NC}(=\mathrm{O}) \mathrm{COc} 1 \mathrm{cccnc} 1$

$\mathrm{Nc} 1[\mathrm{nH}] \mathrm{c}(=\mathrm{O})[\mathrm{nH}] \mathrm{c} 1-\mathrm{c} 1 \mathrm{noc}(\mathrm{C} 2 \mathrm{CCc} 3 \operatorname{ccccc} 3 \mathrm{C} 2) \mathrm{n} 1$

Fc1nnnn1-n1nncc1-n1nnnc1CCc1ccsc1

$[\mathrm{NH} 2+][\mathrm{C} @ @ \mathrm{H}](\mathrm{c} 1 \mathrm{ncns}) \mathrm{C} 1 \mathrm{CSc} 2 \mathrm{ncccc} 2 \mathrm{O} 1$

$\mathrm{Nc} 1[\mathrm{nH}] \mathrm{c}(=\mathrm{O})[\mathrm{nH}] \mathrm{c} 1-\mathrm{c} 1 \mathrm{ncnc} 2[\mathrm{nH}] \mathrm{c} 3 \mathrm{c}(\mathrm{c} 12) \mathrm{CCOC} 3(\mathrm{O}) \mathrm{CC}(=\mathrm{O}) \mathrm{O}$

$\mathrm{Nc} 1 \mathrm{nnc}(\mathrm{Oc} 2 \mathrm{c}(\mathrm{N})[\mathrm{nH}] \mathrm{c} 3 \mathrm{nccc} 23) \mathrm{s} 1$

$\mathrm{Nc} 1[\mathrm{nH}] \mathrm{c}(=\mathrm{O})[\mathrm{nH}] \mathrm{c} 1-\mathrm{c} 1 \mathrm{nn}(-\mathrm{c} 2 \mathrm{nc}[\mathrm{nH}] \mathrm{c} 2-\mathrm{c} 2 \mathrm{ccccc} 2) \mathrm{c} 2 \mathrm{c} 1 \mathrm{C}(=\mathrm{O}) \mathrm{CC}(\mathrm{CC}(=\mathrm{O}) \mathrm{O}) \mathrm{OC} 2=\mathrm{O}$

$\mathrm{Nc} 1[\mathrm{nH}] \mathrm{c} 2 \mathrm{ccccc} 2 \mathrm{c} 1 \mathrm{COc} 1 \mathrm{ccc} 2 \mathrm{c}(\mathrm{c} 1) \mathrm{nnn} 2-\mathrm{n} 1 \mathrm{nnnn} 1 \mathrm{~F}$

$\mathrm{Nc} 1[\mathrm{nH}] \mathrm{c}(=\mathrm{O})[\mathrm{nH}] \mathrm{c} 1 \mathrm{O}[\mathrm{C} @ @ \mathrm{H}] 1 \mathrm{NC}(=\mathrm{O}) \mathrm{c} 2 \mathrm{cccc}(\mathrm{Cl}) \mathrm{c} 2 \mathrm{~S} 1$

$\mathrm{CCn} 1 \mathrm{nnn} 1 \mathrm{C}[\mathrm{C} @ @ \mathrm{H}]([\mathrm{NH} 2+]) \mathrm{c} 1 \mathrm{nc} 2 \mathrm{nccc}[\mathrm{c}-] 2 \mathrm{n} 1$

Fc1nnnn1-n1nnc(C2(N3CC(c4ccccc4)CN3)CC2)n1

$\mathrm{O}=\mathrm{c} 1 \mathrm{c}(-\mathrm{n} 2 \mathrm{nnnn} 2 \mathrm{~F}) \mathrm{cc}(\mathrm{Nc} 2 \mathrm{nnnn} 2 \mathrm{Cc} 2 \mathrm{ccccc} 2) \mathrm{c} 2 \mathrm{cnc}(\mathrm{Cl}) \mathrm{cn} 12$

$\mathrm{Nc} 1[\mathrm{nH}] \mathrm{c}(=\mathrm{O})[\mathrm{nH}] \mathrm{c} 1 \mathrm{OC}[\mathrm{C} @ @ \mathrm{H}] 1 \mathrm{CCO}[\mathrm{C} @ \mathrm{H}](\mathrm{O})[\mathrm{C} @ \mathrm{H}](\mathrm{O})[\mathrm{C} @ \mathrm{H}] 1 \mathrm{O}$

$[\mathrm{NH} 2+][\mathrm{C} @ @ \mathrm{H}](\mathrm{c} 1 \mathrm{ncccn} 1) \mathrm{c} 1 \mathrm{cnc}(\mathrm{Oc} 2 \mathrm{ccccc} 2 \mathrm{~F}) \mathrm{s} 1$

Fc1nnnn1-n1nncc1Nc1ccc2onc3c2c1CCC3

$[\mathrm{NH} 2+][\mathrm{C} @ @ \mathrm{H}](\mathrm{c} 1 \mathrm{ncc}[\mathrm{nH}] 1) \mathrm{c} 1 \mathrm{nc} 2 \mathrm{ccccc} 2 \mathrm{c}(=\mathrm{O}) \mathrm{n} 1-\mathrm{c} 1 \mathrm{ccccc} 1$ 
$[\mathrm{NH} 2+][\mathrm{C} @ \mathrm{H}](\mathrm{C} 1=\mathrm{CCCc} 2 \mathrm{ccccc} 21) \mathrm{c} 1 \mathrm{nccc} 2[\mathrm{nH}] \mathrm{nnc12}$

$\mathrm{Nc} 1[\mathrm{nH}] \mathrm{c}(=\mathrm{O})[\mathrm{nH}] \mathrm{c} 1-\mathrm{c} 1 \mathrm{csc}(\mathrm{Nc} 2 \mathrm{ccc}(\mathrm{F}) \mathrm{cc} 2) \mathrm{n} 1$

$\mathrm{O}=\mathrm{C}(\mathrm{NN}[\mathrm{C} @ \mathrm{H}](\mathrm{Cc} 1 \mathrm{ccccc} 1)[\mathrm{C} @ @ \mathrm{H}](\mathrm{O}) \mathrm{Nc} 1 \mathrm{cnnn} 1-\mathrm{n} 1$ nnnc1F $)$ c1ccnc2ccccc12

$[\mathrm{NH} 2+][\mathrm{C} @ \mathrm{H}](\mathrm{NCc} 1 \mathrm{ccc} 2 \mathrm{c}(\mathrm{c} 1) \mathrm{OCC} 2) \mathrm{c} 1 \mathrm{nc}(-\mathrm{c} 2 \mathrm{ccc}(\mathrm{Br}) \mathrm{cc} 2) \mathrm{no} 1$

$\mathrm{NC}(=\mathrm{O}) \mathrm{SCN}(\mathrm{Nc} 1 \mathrm{cnnn} 1-\mathrm{n} 1 \mathrm{nnn}$ 1F $)$ c1cccc 1

$\mathrm{Nc} 1[\mathrm{nH}] \mathrm{nnc} 1 \mathrm{C}=\mathrm{Nc} 1 \mathrm{nc}(\mathrm{F}) \operatorname{cc}(\mathrm{Cc} 2 \mathrm{ccccc} 2) \mathrm{n} 1$

$\mathrm{Nc} 1[\mathrm{nH}] \mathrm{c}(=\mathrm{O})[\mathrm{nH}] \mathrm{c} 1 \mathrm{O}[\mathrm{CH}] \mathrm{c} 1 \mathrm{ccc}(-\mathrm{n} 2 \mathrm{nnnn} 2) \mathrm{c}(\mathrm{C}(\mathrm{F})(\mathrm{F}) \mathrm{F}) \mathrm{c} 1$

Fc1nnnn1-c1nn[n-]c1OCSCOCc1 1 sc $(-\mathrm{c} 2 \mathrm{ccc}(\mathrm{Cl}) \mathrm{cc} 2) \mathrm{n} 1$

Fc1ncccc1-c1nc([CH]c2nnccc2-n2nnnc2F)ns1

$\mathrm{O}=\mathrm{C}(\mathrm{Oc} 1 \mathrm{ncsc} 1 /[\mathrm{O}+]=\mathrm{c} 1 / \mathrm{oc}(=\mathrm{O})[\mathrm{nH}] \mathrm{s} 1) \mathrm{c} 1 \mathrm{ccccc} 1$

$[\mathrm{NH} 2+][\mathrm{C} @ @ \mathrm{H}](\mathrm{c} 1 \mathrm{nc} 2 \mathrm{ncccn} 2 \mathrm{c} 1 \mathrm{~F}) \mathrm{c} 1 \mathrm{nnnn} 1 \mathrm{Cc} 1 \mathrm{cccs} 1$

$\mathrm{Nc} 1[\mathrm{nH}] \mathrm{c}(=\mathrm{O})[\mathrm{nH}] \mathrm{c1O}[\mathrm{CH}] \mathrm{c} 1 \mathrm{ccc}(\mathrm{NC}(=\mathrm{O}) \mathrm{NCc} 2 \mathrm{cnc}[\mathrm{nH}] 2) \mathrm{cc} 1$

$\mathrm{Nc} 1[\mathrm{nH}] \mathrm{c}(=\mathrm{O})[\mathrm{nH}] \mathrm{c} 1-\mathrm{c} 1 \mathrm{c}[\mathrm{nH}] \mathrm{c} 2 \mathrm{c}(\mathrm{C} 3 \mathrm{CC}=\mathrm{NC} 3=\mathrm{O}) \mathrm{cccc} 12$

$\mathrm{Nc} 1[\mathrm{nH}] \mathrm{c}(=\mathrm{O})[\mathrm{nH}] \mathrm{c} 1 \mathrm{O}[\mathrm{CH}] \mathrm{c} 1 \mathrm{ccccc} 1 \mathrm{NS}(=\mathrm{O})(=\mathrm{O}) \mathrm{C} 1 \mathrm{CC} 1$

$\mathrm{Nc} 1[\mathrm{nH}] \mathrm{c}(=\mathrm{O})[\mathrm{nH}] \mathrm{c} 1 \mathrm{OCCC} 1(\mathrm{~S}(=\mathrm{O})(=\mathrm{O}) \mathrm{c} 2 \mathrm{ccc}(\mathrm{Cl}) \mathrm{cc} 2) \mathrm{CCCCC} 1$

$\mathrm{Nc} 1[\mathrm{nH}] \mathrm{c}(=\mathrm{O})[\mathrm{nH}] \mathrm{c} 1-\mathrm{c} 1 \mathrm{nsc}(\mathrm{S}(=\mathrm{O})(=\mathrm{O}) \mathrm{NCc} 2 \mathrm{ccc}(\mathrm{Br}) \mathrm{cc} 2) \mathrm{n} 1$

$[\mathrm{NH} 2+][\mathrm{C} @ \mathrm{H}](\mathrm{Oc} 1 \mathrm{ccccc} 1) \mathrm{c} 1 \mathrm{nc} 2 \mathrm{ncccc} 2 \mathrm{n} 1-\mathrm{c} 1 \mathrm{noc}(=\mathrm{O})[\mathrm{nH}] 1$

$\mathrm{O}=\mathrm{C} 1 \mathrm{Nc} 2 \mathrm{cc}(\mathrm{Cl}) \operatorname{ccc} 2 \mathrm{C} 1[\mathrm{NH}+] \mathrm{Cc} 1 \mathrm{cnnn} 1-\mathrm{n} 1 \mathrm{nnn} n 1 \mathrm{~F}$

$\mathrm{Nc} 1[\mathrm{nH}] \mathrm{c}(=\mathrm{O})[\mathrm{nH}] \mathrm{c} 1-\mathrm{c} 1 \mathrm{csc}(\mathrm{Nc} 2 \mathrm{noc} 3 \mathrm{c} 2 \mathrm{CCCC} 3) \mathrm{c} 1$

$\mathrm{O}=\mathrm{C}(\mathrm{NCCn} 1 \mathrm{c}(=\mathrm{O}) \mathrm{nnn} 1-\mathrm{n} 1 \mathrm{nnn} \mathrm{c} 1 \mathrm{~F}) \mathrm{Nc} 1 \mathrm{ccccc} 1$

$\mathrm{O}=\mathrm{c} 1 \mathrm{ccn}(\mathrm{Cc} 2 \mathrm{ccccc} 2) \mathrm{c}(\mathrm{Oc} 2 \mathrm{c}(\mathrm{O}) \operatorname{noc} 2 \mathrm{O}) \mathrm{c} 1$

$\mathrm{Nc} 1[\mathrm{nH}] \mathrm{c}(=\mathrm{O})[\mathrm{nH}] \mathrm{c} 1-\mathrm{c} 1 \mathrm{csc}(\mathrm{Nc} 2 \mathrm{nn}(\mathrm{C} 3 \mathrm{CCCCC} 3) \mathrm{c} 3 \mathrm{cc}[\mathrm{nH}] \mathrm{c} 23) \mathrm{n} 1$

$\mathrm{O}=\mathrm{C}(\mathrm{NCc} 1 \mathrm{ccccc} 1) \mathrm{N}(\mathrm{c} 1 \mathrm{nnn}(-\mathrm{n} 2 \mathrm{nnnn} 2 \mathrm{~F}) \mathrm{n} 1) \mathrm{C} 1 \mathrm{CCNCc} 2 \mathrm{ccccc} 21$ $\mathrm{O}=\mathrm{C}(\mathrm{Cl}) \mathrm{ONc} 1 \mathrm{cnnn} 1-\mathrm{n} 1 \mathrm{nnnc} 1 \mathrm{~F}$

Oc1oncc1N[C@@H](c1nnc(-c2ccccc2)o1)C1CC1

$\mathrm{Nc} 1[\mathrm{nH}] \mathrm{c}(=\mathrm{O})[\mathrm{nH}] \mathrm{c} 1 \mathrm{O}[\mathrm{C} @ \mathrm{H}] 1 \mathrm{SCC} 2(\mathrm{NC} 1=\mathrm{O}) \mathrm{C}(\mathrm{O})[\mathrm{C} @ @ \mathrm{H}](\mathrm{O})[\mathrm{C} @ @ \mathrm{H}] 2 \mathrm{O}$

$\mathrm{Nc} 1[\mathrm{nH}] \mathrm{c}(=\mathrm{O})[\mathrm{nH}] \mathrm{c} 1 \mathrm{O}[\mathrm{C} @ \mathrm{H}] 1 \mathrm{C}[\mathrm{C} @ @ \mathrm{H}] 2 \mathrm{OC}[\mathrm{C} @ @] 21 \mathrm{CNCCCC}(=\mathrm{O}) \mathrm{O}$

$\mathrm{Nc} 1[\mathrm{nH}] \mathrm{c}(=\mathrm{O})[\mathrm{nH}] \mathrm{c} 1 \mathrm{OC}(\mathrm{CNC}(=\mathrm{O}) \mathrm{c} 1 \mathrm{ccc}(\mathrm{Cl}) \mathrm{c}([\mathrm{N}+](=\mathrm{O})[\mathrm{O}-]) \mathrm{c} 1) \mathrm{Cc} 1 \mathrm{ccccc} 1$

$[\mathrm{NH} 2+] \mathrm{C}([\mathrm{NH}+]=\mathrm{CC}(=\mathrm{O}) \mathrm{O}) \mathrm{C}(=\mathrm{O}) \mathrm{c} 1 \mathrm{ccc}(\mathrm{O}) \mathrm{cc} 1$

$\mathrm{Nc} 1[\mathrm{nH}] \mathrm{c}(=\mathrm{O})[\mathrm{nH}] \mathrm{c} 1 \mathrm{OCC}(=\mathrm{O}) \mathrm{c} 1 \mathrm{sc}(\mathrm{Nc} 2 \mathrm{ccccc} 2) \mathrm{nc} 1-\mathrm{c} 1 \mathrm{cccc} 1$

$\mathrm{NNc} 1 \mathrm{nnnn} 1 \mathrm{C}(\mathrm{C}(=\mathrm{O}) \mathrm{O}) \mathrm{c} 1 \mathrm{ccc}(\mathrm{F}) \mathrm{cc} 1$

$\mathrm{O}=\mathrm{C}(\mathrm{c} 1 \mathrm{ccco} 1)[\mathrm{C} @ @ \mathrm{H}](\mathrm{COc} 1 \mathrm{c}(\mathrm{O}) \operatorname{noc} 1 \mathrm{O}) \mathrm{Nc} 1 \mathrm{ccccc} 1$

$\mathrm{O}=\mathrm{C} 1 \mathrm{NS} / \mathrm{C} 1=[\mathrm{NH}+] / \mathrm{c} 1[\mathrm{nH}]$ nnc1-c1 $1 \mathrm{ccccc} 1$

$[\mathrm{NH} 2+][\mathrm{C} @ @ \mathrm{H}](\mathrm{c} 1 \mathrm{ncn}[\mathrm{nH}] 1)[\mathrm{C} @ \mathrm{H}](\mathrm{O})[\mathrm{C} @ @ \mathrm{H}] 1 \mathrm{Oc} 2 \mathrm{ccccc} 2 \mathrm{C}[\mathrm{C} @ @ \mathrm{H}] 1 \mathrm{C}(=\mathrm{O})[\mathrm{O}-]$ $\mathrm{O}=\mathrm{C}(\mathrm{N}[\mathrm{C} @ @ \mathrm{H}](\mathrm{Cc} 1 \mathrm{c}[\mathrm{nH}] \mathrm{c} 2 \mathrm{ccccc} 12)[\mathrm{NH} 2+] \mathrm{Cc} 1 \mathrm{cnnn} 1-\mathrm{n} 1 \mathrm{nnnn} 1 \mathrm{~F}) \mathrm{c} 1 \mathrm{nc}(\mathrm{Cl}) \mathrm{ccc} 1 \mathrm{~F}$

$\mathrm{Nc} 1[\mathrm{nH}] \mathrm{c}(=\mathrm{O})[\mathrm{nH}] \mathrm{c} 1-\mathrm{c} 1 \mathrm{c}[\mathrm{nH}] \mathrm{c}(=\mathrm{O}) \mathrm{n}(\mathrm{Cc} 2 \mathrm{ccccc} 2) \mathrm{c} 1=\mathrm{O}$

$\mathrm{Nc} 1[\mathrm{nH}] \mathrm{c}(=\mathrm{O})[\mathrm{nH}] \mathrm{c} 1 \mathrm{OC} 1=\mathrm{Nc} 2 \mathrm{n}[\mathrm{nH}] \mathrm{c}(\mathrm{Cl}) \mathrm{c} 2 \mathrm{C} 1=\mathrm{O}$

$\mathrm{N} \# \mathrm{Cc} 1 \mathrm{cccc}(\mathrm{NCc} 2 \mathrm{nc}(-\mathrm{c} 3[\mathrm{nH}] \mathrm{c}(=\mathrm{O})[\mathrm{nH}] \mathrm{c} 3 \mathrm{~N}) \mathrm{no} 2) \mathrm{c} 1$

$\mathrm{Nc} 1[\mathrm{nH}] \mathrm{c}(=\mathrm{O})[\mathrm{nH}] \mathrm{c} 1-\mathrm{c} 1 \mathrm{csc}(\mathrm{Nc} 2 \mathrm{nncn} 2-\mathrm{c} 2 \mathrm{cccc}(\mathrm{C}(\mathrm{F})(\mathrm{F}) \mathrm{F}) \mathrm{c} 2) \mathrm{n} 1$

$\mathrm{Nc} 1[\mathrm{nH}] \mathrm{c}(=\mathrm{O})[\mathrm{nH}] \mathrm{c} 1 \mathrm{OCc} 1 \mathrm{ncc} 2 \mathrm{cc}(\mathrm{F}) \mathrm{cc}(\mathrm{F}) \mathrm{c} 2 \mathrm{n} 1$

$[\mathrm{NH} 2+][\mathrm{C} @ @ \mathrm{H}](\mathrm{c} 1 \mathrm{ncnc}(\mathrm{Br}) \mathrm{n} 1)[\mathrm{C} @ @ \mathrm{H}] 1 \mathrm{c} 2 \mathrm{ccccc} 2-\mathrm{n} 2 \mathrm{ccnc} 21$

Fc1nnnn1-n1nnc(N(Cc2cccnc2)NCc2ncccn2)n1

$\mathrm{O}=\mathrm{C} 1 \mathrm{NN}(\mathrm{n} 2 \mathrm{nnnc} 2 \mathrm{~F}) \mathrm{N}=\mathrm{NN} 1[\mathrm{C} @ \mathrm{H}] 1 \mathrm{C}[\mathrm{CH}][\mathrm{CH}] \mathrm{C}[\mathrm{NH}+] 1 \mathrm{Cc} 1 \mathrm{ccc}(\mathrm{F}) \mathrm{cc} 1$

Oc1noc $(\mathrm{O}) \mathrm{c} 1 \mathrm{OCNc} 1 \mathrm{n}[\mathrm{n}-][\mathrm{n}+](\mathrm{CCCc} 2 \mathrm{ccccc} 2) \mathrm{c} 1 \mathrm{O}$

$\mathrm{O}=\mathrm{c} 1 \mathrm{nnn}(-\mathrm{n} 2 \mathrm{nnnc} 2 \mathrm{~F}) \mathrm{c} 2 \mathrm{c}(-\mathrm{c} 3 \mathrm{ccccc} 3 \mathrm{~F}) \mathrm{oc}(\mathrm{C} 3 \mathrm{CC} 3) \mathrm{c} 12$

$[\mathrm{NH} 2+][\mathrm{C} @ @ \mathrm{H}](\mathrm{c} 1 \mathrm{ncns}) \mathrm{C}(\mathrm{Oc} 1 \mathrm{nnn}[\mathrm{nH}] 1) \mathrm{C} 1 \mathrm{CCCCC} 1$

Fc1nnnn1-n1nnc(COCc2ccno2)c1OCc1cccc1

$\mathrm{Nc} 1[\mathrm{nH}] \mathrm{c}(=\mathrm{O})[\mathrm{nH}] \mathrm{c1OC}(\mathrm{COC}=\mathrm{O}) \mathrm{NC}(=\mathrm{O}) \mathrm{c} 1 \mathrm{ccccc} 1$

$\mathrm{Nc} 1[\mathrm{nH}] \mathrm{c} 2 \mathrm{ncccc} 2 \mathrm{c} 1 \mathrm{~N}=\mathrm{Nc} 1 \mathrm{nccs} 1$

$\mathrm{Nc} 1[\mathrm{nH}] \mathrm{c}(=\mathrm{O})[\mathrm{nH}] \mathrm{c} 1 \mathrm{OC} 1(\mathrm{c} 2 \mathrm{n}[\mathrm{nH}] \mathrm{c} 3 \mathrm{c}(-\mathrm{c} 4 \mathrm{nc} 5 \mathrm{ccccc} 5[\mathrm{nH}] 4) \mathrm{cccc} 23) \mathrm{COC} 1$

$\mathrm{OC} 1=\mathrm{NN}=\mathrm{NC}(\mathrm{Oc} 2 \operatorname{noc}(\mathrm{O}) \mathrm{c} 2 \mathrm{O}) \mathrm{C} 1 \mathrm{Cc} 1 \mathrm{ccccc} 1$

$\mathrm{Nc} 1[\mathrm{nH}] \mathrm{c}(=\mathrm{O})[\mathrm{nH}] \mathrm{c1}-\mathrm{c} 1 \mathrm{noc}(\mathrm{CNCCO}) \mathrm{n} 1$

$\mathrm{Nc} 1[\mathrm{nH}] \mathrm{c}(=\mathrm{O})[\mathrm{nH}] \mathrm{c} 1 \mathrm{OC}(=\mathrm{S}) \mathrm{Cc} 1 \mathrm{nnnn} 1 \mathrm{Cc} 1 \mathrm{cccnc} 1$

$[\mathrm{NH} 2+][\mathrm{C} @ \mathrm{H}](\mathrm{c} 1 \mathrm{nnn}(-\mathrm{c} 2 \mathrm{ccccc} 2) \mathrm{n} 1) \mathrm{c} 1 \mathrm{nc} 2 \mathrm{cccnc} 2 \mathrm{~s} 1$ 


\begin{tabular}{|c|c|}
\hline & \\
\hline $\operatorname{Nc1}[\mathrm{nH}] \mathrm{c}(=\mathrm{O})[\mathrm{nH}] \mathrm{c1OC}[\mathrm{C} @ \mathrm{H}]([\mathrm{O}-])[\mathrm{C} @ \mathrm{H}](\mathrm{Cc} 1 \mathrm{ccccc} 1) \mathrm{NC}(=\mathrm{O}) \mathrm{NCc} 1 \mathrm{ccccc} 1$ & -5.300 \\
\hline $\begin{array}{l}\mathrm{Nc} 1[\mathrm{nH}] \mathrm{c}(=\mathrm{O})[\mathrm{nH}] \mathrm{c} 1 \mathrm{OC}(=\mathrm{S}) \mathrm{CNC} 1=\mathrm{NC}(=\mathrm{O}) \mathrm{CC} 1 \\
\mathrm{Nc} 1[\mathrm{nH}] \mathrm{c}(=\mathrm{O})[\mathrm{nH}] \mathrm{c} 1 \mathrm{OC}[\mathrm{C} @ \mathrm{H}](\mathrm{N}) \mathrm{COC}[\mathrm{C} @ @ \mathrm{H}] 1 \mathrm{CC}[\mathrm{C} @ \mathrm{H}] 2 \mathrm{CC} 21\end{array}$ & -4.800 \\
\hline $\begin{array}{c}\mathrm{Nc} 1[\mathrm{nH}] \mathrm{c}(=\mathrm{O})[\mathrm{nH}] \mathrm{c} 1 \mathrm{OC}[\mathrm{C} @ \mathrm{H}](\mathrm{N}) \mathrm{COC}[\mathrm{C} @ @ \mathrm{H}] 1 \mathrm{CC}[\mathrm{C} @ \mathrm{H}] 2 \mathrm{CC} 21 \\
\text { Fc1nnnn1-n1nncc1-n1nnc(N=[NH+]Cc2ccc(Br)cc2)n1 }\end{array}$ & -4.800 \\
\hline Fc1nnnn1-n1nncc1-n1nnc(N=[NH+]Cc2ccc(Br)cc2)n1 & -5.800 \\
\hline $\mathrm{O}=\mathrm{C}(\mathrm{O}) \mathrm{CC}(\mathrm{C}(=\mathrm{O}) \mathrm{c} 1 \mathrm{cccc}([\mathrm{N}+](=\mathrm{O})[\mathrm{O}-]) \mathrm{c} 1) \mathrm{N}(\mathrm{c} 1 \mathrm{cccc}(\mathrm{O}) \mathrm{c} 1) \mathrm{P}(=\mathrm{O})([\mathrm{O}-])[\mathrm{O}-]$ & -4.800 \\
\hline $\begin{array}{c}{[\mathrm{NH} 2+][\mathrm{C} @ @ \mathrm{H}](\mathrm{c} 1 \mathrm{nc} 2 \mathrm{nccc}[\mathrm{c}-[2 \mathrm{n} 1) \mathrm{c} 1 \mathrm{cc}(\mathrm{Cl}) \mathrm{ccc} 1-\mathrm{c} 1 \mathrm{ccccc} 1} \\
[\mathrm{NH} 2+][\mathrm{C} @ @ \mathrm{H}](\mathrm{c} 1 \mathrm{n} c[\mathrm{nH}])[\mathrm{C} @ @ \mathrm{H}] 1 \mathrm{c} 2 \mathrm{cc}(\mathrm{NC}=\mathrm{O}) \mathrm{c} 3 \mathrm{ccccc} 3) \mathrm{cc} 2-\mathrm{c} 2 \mathrm{nn}[\mathrm{nH}] \mathrm{c} 21\end{array}$ & -4.800 \\
\hline$[\mathrm{NH} 2+][\mathrm{C} @ @ \mathrm{H}](\mathrm{c} 1 \mathrm{ncc}[\mathrm{nH}] 1)[\mathrm{C} @ @ \mathrm{H}] 1 \mathrm{c} 2 \mathrm{cc}(\mathrm{NC}(=\mathrm{O}) \mathrm{c} 3 \mathrm{ccccc} 3) \mathrm{ccc} 2-\mathrm{c} 2 \mathrm{nn}[\mathrm{nH}] \mathrm{c} 21$ & -5.400 \\
\hline $\begin{array}{l}\text { Fc1nnnn1-n1nnc(CCn2ncnn2)c1CCc1cccc1 } \\
{[\mathrm{NH} 2+][\mathrm{C} @ @ H](\mathrm{c} 1 \mathrm{ncno})[\mathrm{NH}+1 \mathrm{CC} 1 \mathrm{c} 1 \mathrm{ccccc} 1 \mathrm{C}(\mathrm{F})(\mathrm{F}) \mathrm{F}}\end{array}$ & -5.600 \\
\hline$[\mathrm{NH} 2+][\mathrm{C} @ @ \mathrm{H}](\mathrm{c} 1 \mathrm{ncno1})[\mathrm{NH}+] 1 \mathrm{CC} 1 \mathrm{c} 1 \mathrm{ccccc} 1 \mathrm{C}(\mathrm{F})(\mathrm{F}) \mathrm{F}$ & -4.700 \\
\hline$[\mathrm{NH} 2+][\mathrm{C} @ @ \mathrm{H}](\mathrm{c} 1 \mathrm{nccs} 1)[\mathrm{C} @ @ \mathrm{H}] 1 \mathrm{OS}(=\mathrm{O})(=\mathrm{O})[\mathrm{C} @] 12 \mathrm{Cc} 1 \mathrm{c}(\mathrm{Br}) \operatorname{cncc1O} 2$ & -4.600 \\
\hline $\mathrm{Nc} 1[\mathrm{nH}] \mathrm{c}(=\mathrm{O})[\mathrm{nH}] \mathrm{c} 1-\mathrm{c} 1 \mathrm{c}(-\mathrm{c} 2 \mathrm{cccs} 2) \mathrm{nnn} 1-\mathrm{c} 1 \mathrm{ccccc} 1$ & -5.100 \\
\hline $\mathrm{O}=\mathrm{C} 1 / \mathrm{C}(=\mathrm{N} / \mathrm{S}(=\mathrm{O})(=\mathrm{O}) \mathrm{n} 2 \mathrm{cc}(\mathrm{O}) \mathrm{c}(\mathrm{O}) \mathrm{c} 2 \mathrm{O}) \mathrm{NC} 12 \mathrm{CCCC} 2$ & -5.100 \\
\hline $\mathrm{Nc} 1[\mathrm{nH}] \mathrm{c}(=\mathrm{O})[\mathrm{nH}] \mathrm{c} 1 \mathrm{O}[\mathrm{C} @ @ \mathrm{H}] 1 \mathrm{CCc} 2 \mathrm{c}(\mathrm{cc}(\mathrm{Cl}) \mathrm{nc} 2 \mathrm{NCc} 2 \mathrm{ccccc} 2) \mathrm{S} 1$ & -5.200 \\
\hline Nc1nc(NC[C@@H]2CCCO2)c2cc(-c3cscc3-c3[nH]c(=O)[nH]c3N)cnc2n1 & -5.500 \\
\hline $\mathrm{Nc} 1[\mathrm{nH}] \mathrm{c}(=\mathrm{O})[\mathrm{nH}] \mathrm{c} 1 \mathrm{O}[\mathrm{C} @ \mathrm{H}] 1 \mathrm{SN}([\mathrm{C} @ @ \mathrm{H}](\mathrm{c} 2 \mathrm{ccccc} 2) \mathrm{c} 2 \mathrm{c}(\mathrm{Cl}) \mathrm{cccc} 2 \mathrm{Cl}) \mathrm{C} 1 \mathrm{O}$ & -5.500 \\
\hline$[\mathrm{NH} 2+][\mathrm{C} @ @ \mathrm{H}](\mathrm{c} 1 \mathrm{ncno1})[\mathrm{C} @ \mathrm{H}](\mathrm{Cc} 1 \mathrm{c}[\mathrm{nH}] \mathrm{c} 2 \mathrm{ccccc} 12) \mathrm{c} 1 \mathrm{nc} 2 \mathrm{ccc}([\mathrm{N}+](=\mathrm{O})[\mathrm{O}-]) \mathrm{cc} 2[\mathrm{nH}] \mathrm{c} 1=\mathrm{O}$ & -5.300 \\
\hline $\mathrm{O}=\mathrm{C}(\mathrm{CC}[\mathrm{C} @ @ \mathrm{H}] 1 \mathrm{CNC}(\mathrm{c} 2 \mathrm{cnnn} 2-\mathrm{n} 2 \mathrm{nnnc} 2 \mathrm{~F}) \mathrm{N} 1) \mathrm{c} 1 \mathrm{ccccc} 1 \mathrm{Cl}$ & -5.600 \\
\hline $\mathrm{O}=\mathrm{C}(\mathrm{O}[\mathrm{C} @ @ \mathrm{H}] 1[\mathrm{C} @ \mathrm{H}] 2 \mathrm{OC}[\mathrm{C} @ \mathrm{H}](\mathrm{Oc} 3 \mathrm{nc}(\mathrm{Cl}) \mathrm{nn} 3 \mathrm{CCO})[\mathrm{C} @ \mathrm{H}] 2[\mathrm{C} @ \mathrm{H}] 1 \mathrm{O}) \mathrm{c} 1 \mathrm{ccco} 1$ & -4.700 \\
\hline $\mathrm{O}=\mathrm{C} 1 \mathrm{c} 2 \mathrm{ccccc} 2 \mathrm{C} 1 \mathrm{C}(\mathrm{Oc} 1 \mathrm{c}(\mathrm{O})$ noc1O $)$ c1nsc2ncccc12 & -5.300 \\
\hline $\mathrm{Nc} 1[\mathrm{nH}] \mathrm{nnc} 1 \mathrm{CNC}(=\mathrm{O}) \mathrm{c} 1 \mathrm{ccc}(\mathrm{Cl}) \mathrm{cc} 1$ & -5.000 \\
\hline $\mathrm{Cc} 1[\mathrm{nH}] \mathrm{c} 2 \operatorname{ccccc} 2 \mathrm{c} 1 \mathrm{C}(=\mathrm{O}) \mathrm{ON}=\mathrm{CNC}(\mathrm{N}) \mathrm{NC}(=\mathrm{O}) \mathrm{c} 1 \mathrm{ccccc} 1$ & -5.700 \\
\hline $\mathrm{Nc} 1[\mathrm{nH}] \mathrm{c}(=\mathrm{O})[\mathrm{nH}] \mathrm{c} 1-\mathrm{c} 1 \mathrm{ncnc} 2[\mathrm{nH}] \mathrm{c}(\mathrm{C}(\mathrm{N}) \mathrm{c} 3 \mathrm{ccccc} 3) \mathrm{cc} 12$ & -5.500 \\
\hline $\mathrm{Nc} 1[\mathrm{nH}] \mathrm{c}(=\mathrm{O})[\mathrm{nH}] \mathrm{c} 1 \mathrm{OC}(\mathrm{Cc} 1 \mathrm{ccccc} 1)(\mathrm{Cc} 1 \mathrm{ncn}[\mathrm{nH}] 1) \mathrm{C}(=\mathrm{O}) \mathrm{N} 1 \mathrm{CCCC} 1$ & -5.200 \\
\hline $\mathrm{O}=\mathrm{C}(\mathrm{Nc} 1 \mathrm{ccc}(\mathrm{Oc} 2 \mathrm{ccnn} 2-\mathrm{n} 2 \mathrm{nnn} 2 \mathrm{~F}) \mathrm{cc} 1) \mathrm{Nc} 1 \mathrm{cccc} 2 \mathrm{ncncc} 12$ & -5.100 \\
\hline $\mathrm{Nc} 1[\mathrm{nH}] \mathrm{c}(=\mathrm{O})[\mathrm{nH}] \mathrm{c} 1-\mathrm{c} 1 \mathrm{nnn}(-\mathrm{c} 2 \mathrm{ccc}(\mathrm{Br}) \mathrm{c}([\mathrm{N}+](=\mathrm{O})[\mathrm{O}-]) \mathrm{c} 2) \mathrm{n} 1$ & -5.200 \\
\hline $\mathrm{N}[\mathrm{C} @ \mathrm{H}](\mathrm{CCC}(=\mathrm{O})[\mathrm{O}-]) \mathrm{c} 1[\mathrm{nH}] \mathrm{sc} 1[\mathrm{C} @ @ \mathrm{H}]([\mathrm{NH} 2+]) \mathrm{c} 1 \mathrm{ncc}[\mathrm{nH}] 1$ & -4.500 \\
\hline $\mathrm{Nc} 1[\mathrm{nH}] \mathrm{c}(=\mathrm{O})[\mathrm{nH}] \mathrm{c} 1-\mathrm{c} 1 \mathrm{csc}(-\mathrm{c} 2 \mathrm{ccnc}(\mathrm{N}=\mathrm{Nc} 3 \mathrm{nc} 4 \mathrm{ccccc} 4 \mathrm{o} 3) \mathrm{n} 2) \mathrm{n} 1$ & -5.700 \\
\hline$[\mathrm{NH} 2+][\mathrm{C} @ @ \mathrm{H}](\mathrm{c} 1 \mathrm{nc} 2 \mathrm{ncccc} 2[\mathrm{nH}] 1) \mathrm{c} 1 \mathrm{c}[\mathrm{nH}] \mathrm{nc1}-\mathrm{c} 1 \mathrm{ccccc} 1$ & -4.800 \\
\hline$[\mathrm{NH} 2+][\mathrm{C} @ @ \mathrm{H}](\mathrm{c} 1 \mathrm{nc} 2 \mathrm{nccc}[\mathrm{c}-] 2 \mathrm{n} 1)[\mathrm{C} @ \mathrm{H}] 1 \mathrm{CC}(=\mathrm{O}) \mathrm{OC} 1 \mathrm{O}$ & -4.600 \\
\hline Fc1nnnn1-n1nncc1N $[\mathrm{C} @ \mathrm{H}](\mathrm{C}[\mathrm{NH} 2+] \mathrm{c} 1 \mathrm{ccc} n 1) \mathrm{c} 1 \mathrm{ccccc} 1$ & -5.600 \\
\hline Fc1nnnn1-c1nnn(-c2nsc(NC3CCCCCC3)n2)n1 & -5.700 \\
\hline $\mathrm{Nc} 1[\mathrm{nH}] \mathrm{c}(=\mathrm{O})[\mathrm{nH}] \mathrm{c} 1 \mathrm{C} 1=\mathrm{S}(\mathrm{C} 2 \mathrm{CC} 2) \mathrm{C}=\mathrm{C} 1 \mathrm{Nc} 1 \mathrm{nc} 2 \mathrm{scnc} 2 \mathrm{nc} 1-\mathrm{c} 1 \mathrm{ccccc} 1$ & -5.300 \\
\hline $\mathrm{O}=\mathrm{C} 1 \mathrm{Nc} 2 \mathrm{ccccc} 2 \mathrm{C} 1 \mathrm{C}(\mathrm{O}) \mathrm{c} 1 \mathrm{cncc} 2[\mathrm{nH}] \mathrm{nnc} 12$ & -5.200 \\
\hline $\mathrm{Nc} 1[\mathrm{nH}] \mathrm{c}(=\mathrm{O})[\mathrm{nH}] \mathrm{c} 1 \mathrm{OC} 1=\mathrm{CNc} 2 \mathrm{c}(\mathrm{F}) \operatorname{ccc} 2 \mathrm{~N} 1$ & -4.900 \\
\hline $\mathrm{Nc} 1[\mathrm{nH}] \mathrm{nnc} 1 \mathrm{C} 1 \mathrm{C}=\mathrm{C} 2 \mathrm{CCN}(\mathrm{S}(=\mathrm{O})(=\mathrm{O}) \mathrm{C} 3(\mathrm{COc} 4 \operatorname{ccccc} 4) \mathrm{CCCC} 3) \mathrm{CC} 2 \mathrm{~N} 1$ & -5.200 \\
\hline$[\mathrm{NH} 2+][\mathrm{C} @ \mathrm{H}](\mathrm{Cc} 1 \mathrm{nnc}(\mathrm{N}[\mathrm{C} @ \mathrm{H}] 2 \mathrm{CCOc} 3 \mathrm{ccc}(\mathrm{F}) \mathrm{cc} 32) \mathrm{s} 1) \mathrm{c} 1 \mathrm{ncco} 1$ & -5.500 \\
\hline$[\mathrm{NH} 2+][\mathrm{C} @ \mathrm{H}](\mathrm{c} 1 \mathrm{ccc}(\mathrm{F}) \mathrm{cc} 1) \mathrm{c} 1 \mathrm{nc}(-\mathrm{n} 2 \mathrm{ccnn} 2) \mathrm{oc} 1 \mathrm{~N}[\mathrm{C} @ \mathrm{H}](\mathrm{CCO}) \mathrm{c} 1 \mathrm{ccncc} 1$ & -4.900 \\
\hline $\mathrm{N}[\mathrm{C} @ @ \mathrm{H}](\mathrm{OCS}(\mathrm{N})(=\mathrm{O})=\mathrm{O}) \mathrm{c} 1 \mathrm{cccc}(-\mathrm{c} 2 \mathrm{nnc}(\mathrm{Oc} 3 \operatorname{ccnc} 4 \operatorname{ccccc} 34) \mathrm{s} 2) \mathrm{c} 1$ & -5.400 \\
\hline $\mathrm{O}=\mathrm{Nc} 1 \mathrm{nnn}(-\mathrm{n} 2 \mathrm{nnn} \mathrm{n} 2 \mathrm{~F}) \mathrm{c} 1 \mathrm{NCc} 1 \mathrm{cccc} 2 \mathrm{cnccc} 12$ & -5.700 \\
\hline$[\mathrm{NH} 2+][\mathrm{C} @ \mathrm{H}](\mathrm{NC}[\mathrm{C} @ \mathrm{H}] 1 \mathrm{OC}[\mathrm{C} @ @ \mathrm{H}] 1 \mathrm{Cc} 1 \mathrm{ccccc} 1) \mathrm{c} 1 \mathrm{ncno1}$ & -4.800 \\
\hline$[\mathrm{NH} 2+][\mathrm{C} @ \mathrm{H}](\mathrm{C}[\mathrm{C} @ @ \mathrm{H}] 1 \mathrm{NC}(=\mathrm{O}) \mathrm{c} 2 \mathrm{nc}(-\mathrm{c} 3 \mathrm{ccccc} 3)[\mathrm{nH}] \mathrm{c} 21) \mathrm{C} 1=\mathrm{Nc} 2 \mathrm{ccncc} 21$ & -5.500 \\
\hline $\mathrm{Nc1}[\mathrm{nH}] \mathrm{c}(=\mathrm{O})[\mathrm{nH}] \mathrm{c} 1 \mathrm{OCCn} 1 \mathrm{cc}(-\mathrm{c} 2 \mathrm{ccccc} 2 \mathrm{Cl}) \mathrm{nn} 1$ & -5.700 \\
\hline$[\mathrm{NH} 2+][\mathrm{C} @ @ \mathrm{H}](\mathrm{c} 1 \mathrm{nc} 2 \mathrm{ncccn} 2 \mathrm{c} 1-\mathrm{n} 1 \mathrm{nnn}(-\mathrm{-c} 2 \mathrm{ccco} 2) \mathrm{n} 1) \mathrm{C} 1 \mathrm{CC} 1$ & -5.300 \\
\hline $\mathrm{Nc} 1[\mathrm{nH}] \mathrm{c}(=\mathrm{O})[\mathrm{nH}] \mathrm{c} 1 \mathrm{OC}[\mathrm{C} @ \mathrm{H}] 1 \mathrm{COc} 2 \mathrm{cc}(\mathrm{Br}) \mathrm{ccc} 2 \mathrm{~N} 1$ & -5.000 \\
\hline
\end{tabular}




\section{S 6 Graph representation of the hierarchy of chemical fragments}

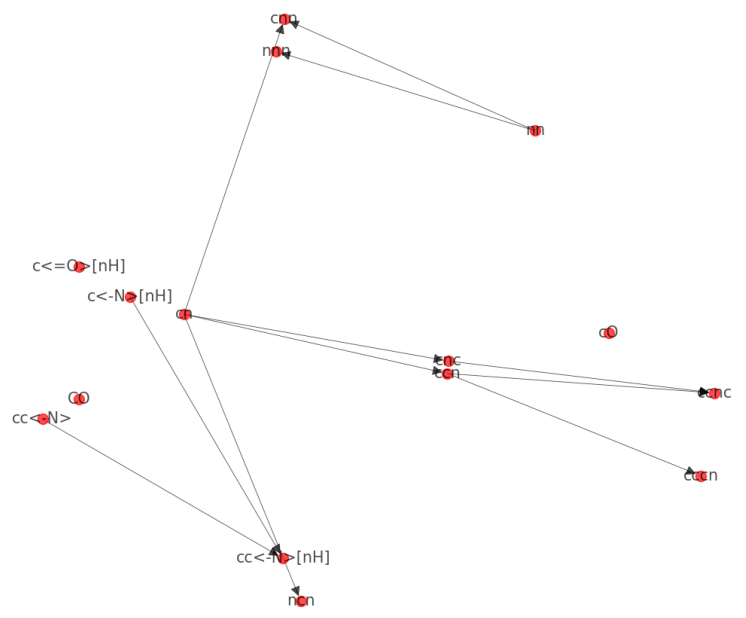

Figure S6: The chemical fragments identified by the molecular fragmenting algorithm in RDKit is represented as a directed graph. The directed edges one fragment to another indicate hierarchy. The terminal nodes then correspond to largest unique fragments. Only the most commonly occuring fragments within the top 200 candidates are shown in this figure for clarity

\section{S $7 \quad$ MTNN predictions for commonly used drugs}

Table S2: S-protein Vina scores for some of the commonly used drugs for treatment for COVID-19 as predicted by our retrained MTNN model

\begin{tabular}{||c|c|c|}
\hline Drug name & S-protein score predicted by MTNN & S-protein score predicted by Auto-docking \\
\hline \hline Remdesivir & -4.67 & -4.90 \\
Nelfinavir & -5.01 & -5.10 \\
Hydroxychloroquine & -4.22 & -4.20 \\
SSAA09E2 & -4.73 & -4.60 \\
K22 & -4.97 & -5.50 \\
GW280264X & -4.35 & -4.80 \\
Dexamethasone & -5.14 & -4.90 \\
Azithromycin & -4.30 & -4.40 \\
Acalabrutinib & -5.12 & -4.50 \\
Baricitinib & -4.66 & -5.10 \\
Ruxolitinib & -4.56 & -5.30 \\
Tofacitinib & -4.85 & -4.80 \\
Lopinavir & -4.68 & -4.10 \\
Ivermectin & -4.54 & -4.60 \\
Oseltamivir & -4.45 & -4.00 \\
Colchicine & -4.44 & -4.50 \\
\hline
\end{tabular}




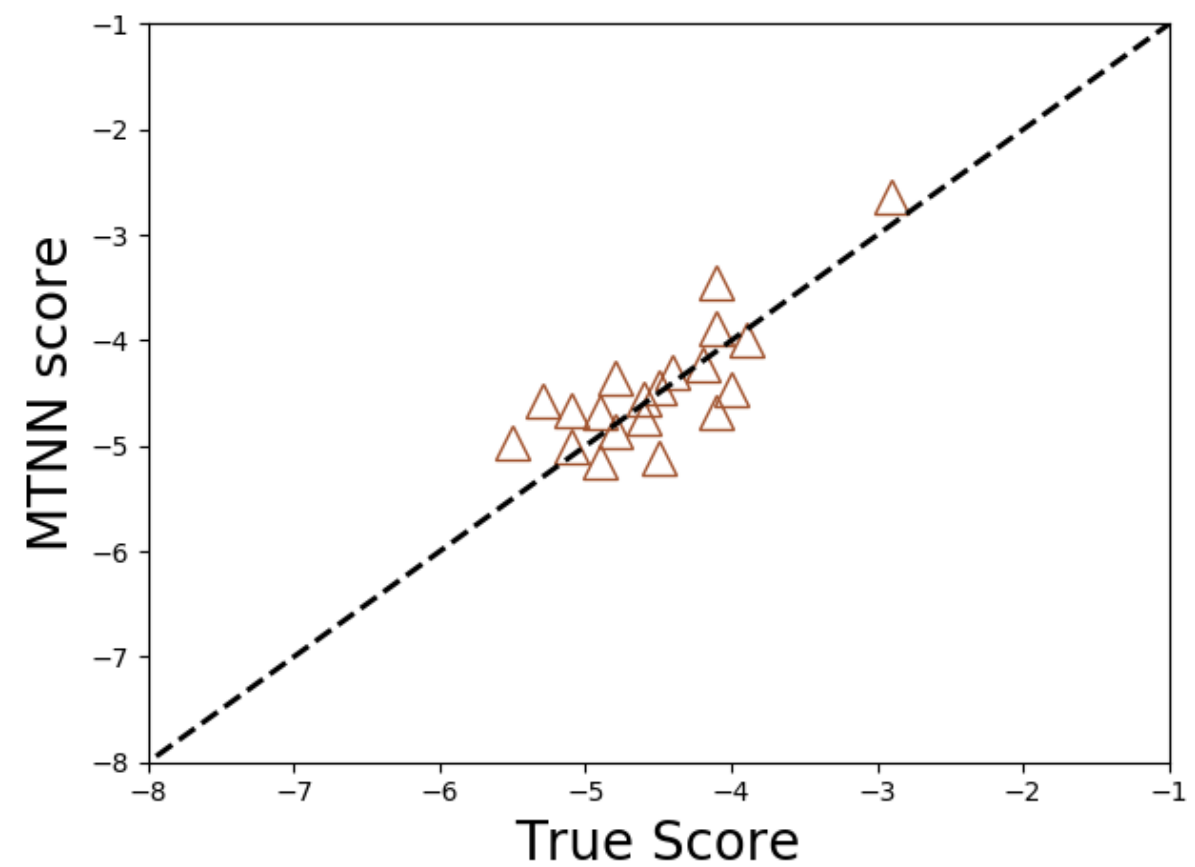

Figure S7: Parity plot of S-protein Vina score predicted by the MTNN. The true scores are obtained from Auto-docking simulations 